\title{
Mild stress stimuli built into a non-immersive virtual environment can elicit actual stress responses
}

\author{
Mohammed Alghamdi ${ }^{a}$, Holger Regenbrecht ${ }^{a}$, Simon Hoermann ${ }^{a, b, c *}$ and Nicola Swain ${ }^{d}$ \\ ${ }^{a}$ Department of Information Science, University of Otago, Dunedin, New Zealand; ${ }^{b}$ Department of Medicine (DSM), University of Otago, \\ Dunedin, New Zealand; 'School of Electrical and Information Engineering, The University of Sydney, Sydney, Australia; ${ }^{\mathrm{d} D e p a r t m e n t}$ of \\ Psychological Medicine, University of Otago, Dunedin, New Zealand
}

\begin{abstract}
The experience of Virtual Reality (VR) can lead to unwanted or wanted psychological stress reactions. Highly immersive VR games for instance utilise extreme, life-threatening, or dangerous situations to achieve those responses from their players. There is also sufficient evidence that in clinical settings and specific situations, such as fear of heights or post-traumatic stress, virtual stimuli can lead to perceived stress for clients. However, there is a gap in research targeting everyday, mild emotional stimuli, which are neither extreme nor specific and which are not presented in an immersive system. To what extent can common stimuli in a non-immersive virtual environment elicit actual stress reactions for its users? We developed a desktop VR system and evaluated it in a study with 54 participants. We could show that virtual stimuli in a common, domestic family environment led to a significant increase in perceived stress as measured by quantitative (self-reports) and qualitative (semi-structured interviews analysed with a General Inductive Approach (GIA)) responses. The results also showed that the introduction of virtual stimuli induced significantly higher levels of perceived workload and sense of presence and led to different physiological reactions. These findings have implications for the design and implementation of non-immersive VR systems.
\end{abstract}

ARTICLE HISTORY

Received 2 March 2016

Accepted 13 March 2017

\section{KEYWORDS}

Virtual Reality; stress; virtual stimuli; exposure; sense of presence

\section{Introduction}

For most of us, standing at the edge of a high cliff is an uncomfortable, even stressful experience. We might get sweaty hands, our heart rate (HR) goes up, we can hardly concentrate on anything else than mastering this situation, and we might even start to panic. These responses to a stressful situation help us to survive or at least to keep us out of danger most of the times. However, some people develop fears which are so severe that they interfere with their normal life and therefore they need help (North, North, and Coble 1998). Mental health professionals often use an adjunct Virtual Reality Exposure Therapy (VRET) approach (Krijn et al. 2004). In VRET, clients are exposed to fearful or stress-evoking virtual stimuli to develop coping strategies for real-life situations (Wiederhold, Bullinger, and Wiederhold 2006). There is an extensive body of research showing that those specific virtual stimuli actually lead to equivalent psychological responses for users (Mühlberger et al. 2007), for instance, eliciting anxiety (e.g. Pertaub, Slater, and Barker 2002; Hartanto et al. 2014; Seinfeld et al. 2016), stress (e.g. Slater et al. 2006; Stetz et al. 2006), and fears (e.g. Diemer et al. 2014; McCall et al. 2015). Apparently, virtual stressors are as effective as real-life stimuli in those extreme situations.

Similarly, when playing highly immersive computer games, people are seeking the experience of stressful situations (amongst other forms of experiences) by getting stimulated with the virtual stressors presented in the games. Those stimuli include dark, claustrophobic spaces, (virtual) life-threatening game characters such as monsters, and, again, high cliffs or dangerous animals, such as spiders (e.g. McCall et al. 2015).

Highly immersive computer games and VRET use similar stimulation techniques to evoke certain psychological responses, such as stress. There are even situations where computer games are used for VRET focusing on significant life changes or scenarios associated with major life events, like with post-traumatic stress disorder research (e.g. Difede and Hoffman 2002; Rizzo et al. 2010; Rizzo et al. 2014); fear of spiders (Garcia-Palacios et al. 2002); fear of cockroaches (e.g. Botella et al. 2005); fear of heights (e.g. Krijn et al. 2004); fear of flying (Rothbaum et al. 1996); and fear of public speaking/social phobia (e.g. Botella et al. 2000). 
However, life is more than just being in specific and extreme situations: at cliffs, at war, in jungles full of spiders, or fighting armed monsters. What about everyday events which make us feel stressed? The too-manythings-at-once situations, the noisy neighbourhood, or our children playing with lighters or knives? While there is no doubt that those situations make us feel stressed in real life (Kanner et al. 1981; Crnic and Greenberg 1990; Kohn and Macdonald 1992), it is unclear whether virtual, everyday stressors would elicit actual stress responses. Are virtual stressors only effective for specialised or extreme situations or will they work for everyday situations as well?

In addition, if those virtual stressors are presented in a highly immersive system, for example, by utilising headmounted displays (HMDs) or surrounding stereo projections, there is little doubt that this immersive experience can lead to actually perceived stress. But, is that true for the more widely used class of virtual reality (VR) systems utilising just a monitor screen? Those non-immersive systems are readily available, are familiar to most people, and are less prone to evoke stress or other emotional responses by their sheer appearance. For example, when putting on a HMD or entering a CAVE, this act in itself will provoke reactions, at least for first-time or occasional users.

Krijn et al. (2004) point out that ' ... VRET should be distinguished from different forms of treatments [...] [for instance] computer-assisted vicarious exposure'. The latter would use a 2D screen but not necessarily a 3D virtual environment (on screen), like in the system suggested and presented in this paper here. They also point out that 'Research is needed to compare VRET with these other computer-mediated treatments'. Our work addresses this by presenting a non-immersive, but nevertheless VR system to be used to evoke psychological responses of stress.

If we can show if and how mild everyday stimuli presented in a common, non-immersive VR system can elicit actual stress responses, then this will influence our decisions on the design of virtual environments, either to avoid stressful experiences for our users or to provide such experiences like in (casual) VR games. Also, would virtually evoked stress responses lead to higher workload and a higher sense of presence for the users? While the higher perceived workload would just confirm our common-sense judgement, a higher sense of presence means that the virtual environment is perceived as a virtual world. A perceived sense of presence is a defining element of VR (Slater 2009).

Based on a scenario around an everyday, domestic family environment, we present a study which shows a positive, significant relationship between virtual, everyday emotional stimuli and stress responses. We also show that workload and presence are significantly affected.

To the best of our knowledge, this research is the first of its kind to demonstrate a relationship between nonextreme, non-specialised, virtual, everyday situations and stress responses; workload measures; and a perceived sense of being in a virtual environment (sense of presence) with a non-immersive VR system.

\section{Related work}

\subsection{Virtual reality}

VR combines three-dimensionality, real-time computer graphics, and interactivity in a way that users feel being part of that environment. VR can appear in a variety of forms depending on the hardware and software being used (Krijn et al. 2004), ranging from non-immersive desktop-monitor systems to so-called CAVE installations, which are highly immersive, mainly due to the surroundedness of the users.

The effectiveness of a virtual environment depends on several elements. The sense of being part of the environment is a defining element. This sense of presence has been investigated and studied by many researchers (e.g. Heeter 1992; Loomis 1992; Sheridan 1992; Barfield et al. 1995), and it can be defined as 'the subjective experience of being in one place or environment [e.g. virtual environment], even when one is physically situated in another' (Witmer and Singer 1998, 225); or as 'a state of consciousness, the (psychological) sense of being in the virtual environment' (Slater and Wilbur 1997, 4). High levels of presence that users experience in the VR environment should make them perceive this environment as a more engaging reality than the surrounding physical world, and consider the environment as a place they have visited rather than just images they have seen (Slater and Wilbur 1997). Thus, participants' behaviours in this VR environment should be consistent with those that would have occurred in real life under similar circumstances (Slater and Wilbur 1997).

Normally, a higher immersive system would lead to higher levels of perceived sense of presence and, therefore, would lead to stronger psychological reactions. Whether less immersive systems are effective in that sense mainly depends on the modes and levels of interactivity and on the architecture and narrative of the virtual environment (Regenbrecht 2000).

\subsection{VR and evoking psychological reactions}

Users can be emotionally affected by virtual environments, even though they know these environments are 
not real (Walshe et al. 2005). There has been research showing that in particular immersive VR environments or stimuli can induce (strong) emotional and psychological reactions in users (Meehan et al. 2003). For example, Meehan et al. (2002) conducted experiments to examine and compare users' physiological reactions when exposed to a non-threatening virtual environment compared with a threatening, stressful one. Their results revealed that a response/change in HR resulted from the VR exposure. González-Franco et al. (2014) used a scenario where a group of participants observed an attack on their virtual body by a knife in an immersive virtual environment. These are examples of extreme and specific situations. Or, for instance, McCall et al. (2015) studied arousal, which is a subjective feeling with strong physiological correlates. During the study, they immersed participants in a threatening VR environment ('Room 101'). Participants were confronted with a series of fearful events such as facing many spiders (crawling down the walls and the ceiling), scary ambient noise (sounds of footsteps and gunshots), blood appearing on the walls and across the view, and several explosions destroying the ground. One of their results showed that this fearful environment was successful in eliciting physiological responses (HR and skin conductance).

A number of studies have shown that virtual human avatars can elicit a type of fear or anxiety similar to what people may experience in real-life social situations (Slater, Pertaub, and Steed 1999; Pertaub, Slater, and Barker 2001; Pertaub, Slater, and Barker 2002; Garau et al. 2005; Slater et al. 2006).

Some previous research have suggested that there is a link between presence and emotions in VR (Pallavicini et al. 2013). Emotions such as fear, anxiety, or stress perceived during immersion were found to be related to presence (Bouchard et al. 2008). Regenbrecht, Schubert, and Friedmann (1998) and Schuemie et al. (2000) found significant associations between the level of fear experienced during immersion and presence. This is supported by Michaud et al. (2004) and Bouchard et al. (2008) who performed experiments manipulating the levels of presence and then examining its influence on anxiety. A comprehensive review of perception and perception on emotional reactions, in particular fears, can be found in Diemer et al. (2015). She and her colleagues developed a new attribution model for presence, including the identification of gaps in research.

Research in the application of non-immersive virtual environments on psychological responses is less numerous, but promising.

A study by Klinger et al. (2005) could show that cognitive behaviour therapy to treat social phobias administered traditionally and using a PC-based VR system showed similar effects. Here, participants sat in front of a large monitor and navigated in the virtual environment using a mouse, arrow keys, or a cyberpuck - a non-immersive set-up, but with specific, clinical stimuli.

The effects of non-immersive VR on phobic (clinical) and non-phobic (healthy control subjects) were investigated by Robillard et al. (2003), showing that anxiety and presence only developed for the phobic participants and that there is a relationship between presence and anxiety. Apparently, exposing people to a non-immersive virtual environment can lead to psychological responses, but this study is leaving the question unanswered whether this also works for non-phobic stimuli and for non-phobic users.

Baños et al. (2004) addressed the level of immersion on presence mediated by emotional elements in the virtual environment. Both immersion and affective content have an impact on presence, but immersion was more relevant for non-emotional versus emotional environments. Hence, it is left unanswered whether a nonimmersive system (less immersion) can elicit (sufficient) psychological responses.

While there is a host of research into VRET, predominantly immersive, but also non- and semi-immersive, and while there is some research in non-immersive virtual exposure with specific stimuli, there seems to be no or only little research into rather common, mild virtual stimuli in non-immersive, desktop-monitor environments.

We are utilising a non-specific, non-clinical, common scenario to investigate this gap in research. Our scenario of postnatal parenting is well defined and with this can be rigorously studied, while at the same time it is common enough to allow for generalisations towards other applications.

Our research is motivated by requests from research and practice in mental health, in particular in addressing postnatal stress and depression, and on how VR can be used to minimise negative experiences after a child is born for the parents. We opted for not tackling the clinical nature of this topic, but rather for taking parenthood as an omnipresent situation worth to serve as an example for our research.

\subsection{Parenting of very young children}

For this targeted scenario situated in a domestic family environment, children play a vital role in experiencing such an environment, including developing stressful responses, in particular in the postnatal period, which is a critical time for every parent, especially a mother. 
During this time, several daily stressors and emotional challenges typically arise.

Most families and parents typically face daily frustrating and difficult events/tasks from child-bearing and caregiving demands (Crnic and Greenberg 1990). For some parents, particularly those who are having children for the first time, this is a new and different environment they need to adapt to. It is expected that all parents have to deal with daily common parenting situations such as being nagged or whined at, responding and resolving siblings' arguments, frequently tidying and cleaning up children's mess, and more of other, similar everyday events (Crnic and Greenberg 1990). Parenting a child can be a difficult task (Crnic and Booth 1991). More challenging children tend to be a source of significant daily stressors (Crnic and Greenberg 1990). With children growing and developing more abilities, they may also show behaviours that are considered by parents as stressful (Crnic and Booth 1991). These daily stressors are sometimes called hassles. Crnic and Greenberg (1990) described them as 'irritating, frustrating, annoying, and distressing demands that to some degree characterize everyday transactions with the environment' (Crnic and Greenberg 1990, 1629). Being in such an environment and dealing with these everyday stressful situations can affect the psychological well-being of the parent. An individual, everyday event might have a small effect in itself; however, the cumulative impact of them over a day, several days, or longer periods can be perceived as stressors for parents and result in significant implications for the functioning of the parents and their families (Crnic and Greenberg 1990). A study by Crnic and Greenberg (1990) reported that parenting stressors are associated with less satisfied parenting and less functional family status. Such stressors are not only putting the children at risk because of the stress, but, in fact, it also affects the whole family system (Patterson 1983). These everyday parenting stressors can be important determinants of parental wellbeing (Crnic and Booth 1991). They also might be a meaningful and more relevant context for conceptualising stress (DeLongis et al. 1982).

In summary, there is a huge body of work on the development of (strong) psychological responses to virtual stimuli, but no work investigates the apparent question of whether everyday situations and stressors presented in a non-immersive VR system can actually elicit stress responses to the users of the VR systems. We are addressing this gap by identifying suitable stressors for a common environment and scenario and by implementing and evaluating these stressors in a desktop-monitor virtual environment context.

\section{Identifying a sample of common everyday stressors for parents}

This part of the research sought to practically identify and evaluate the most common everyday stressors in a scenario around a domestic family environment. To be more specific, the focus was on stressors with the following characteristics: everyday stressors that typically occur for parents in a house environment after having a newborn child.

Three coherent, subsequent phases were designed and employed.

A comprehensive review of related literature was conducted to collect and examine common stressors or stressful events/situations that may affect the psychological well-being of a person. The gathered stressors from this review fall into the following categories: (1) major stressors (e.g. death of spouse, divorce, or fired at work (Holmes and Rahe 1967)); (2) minor everyday stressors (e.g. too many things to do, home maintenance, or preparing meals (Chamberlain and Zika 1990)); and (3) special everyday family and parenting stressors including those after having a new child (e.g. continually cleaning up messes of toys or food, the need to keep a constant eye on where the kids are and what they are doing, and the kids' demand to be entertained or to be played with (Crnic and Greenberg 1990)).

After removing the duplicated stressors, a comprehensive list of 152 stressors was generated from several references.

Relying on one source (e.g. literature review) to gather requirements can result in insufficient data. A deeper knowledge and understanding can be achieved by conducting qualitative research (Cooper, Reimann, and Cronin 2007). Semi-structured interviews were conducted as a second source to identify and explain the most common everyday stressors for parents that typically happen in a home environment after having a newborn child. Six semi-structured interviews were conducted with six domain experts (mental health specialists). All were psychotherapists who are specialised in family, parenting, children, stress, and anxiety problems. The gathered data were analysed and results were added to the findings from the first phase.

It was not feasible nor desirable to implement and simulate all the stressors that were identified in the first two phases in the VR system. Therefore, another evaluation phase was necessary to better define and narrow down the results. A web-based survey was designed to assess the identified stressors based on their level of influence during the postnatal period on parents. The same six psychotherapists were invited 
again to participate in the survey. Their task was to indicate (based on their experience) the extent to which a parent who recently had a newborn child would be stressed in a home environment by the events in the list provided from the previous results. Seven-point Likert-like items were used, anchored with 'Not at all stressed' to 'Extremely stressed'.

The web-based survey resulted in a list of the stressors rated from the most to the least stressful based on the knowledge and experience of the domain experts. Given the scope of this research, and the focus on the everyday stressors, a final evaluation was performed, which excluded (1) all major stressors and (2) stressors that were clearly not relevant to the chosen environment in this research (a house environment).

For the rest of the stressors in the list, the new focus was on the most effective stimuli. The following top 13 most effective everyday stimuli as rated by the domain experts formed the basis for the implemented virtual stressors in the VR system:

(1) Baby crying.

(2) New family member.

(3) Continually cleaning up messes of toys or food.

(4) The need to keep a constant eye on where the kids are and what they are doing.

(5) Too many things to do.

(6) Too many things to do at once.

(7) Too many responsibilities.

(8) Overloaded with family responsibilities.

(9) Too many interruptions.

(10) Experiencing high levels of noise.

(11) Troublesome neighbours.

(12) Unsatisfactory housing conditions.

(13) Housework.

A suitable main task in the virtual environment was also developed from these findings.

The following section explains the system development including the implementation of the virtual stressors driven by the findings above. For the rest of the paper, the word 'stressors' will always refer to the 'common, mild, everyday stimuli that typically occur for parents in a house environment after having a newborn child'. The 13 identified stressors are the sample used in this research.

While the above list is specific to the postnatal period in a domestic family house, they also include representative classes of stressors found in other environments. Interruptions, noise, workload-related stimuli, and so on, happen in almost all domestic, commercial, industrial, or clinical situations.

\section{System design and implementation}

Using Google SketchUp ${ }^{\mathrm{TM}}$ software, a 3D house was modelled to be the virtual environment (Alghamdi et al. 2016). It is an approximately 737 square metre family house consisting of a main living room, kitchen, one bathroom, two bedrooms, and front- and backyards. A collection of 3D off-the-shelf furniture was used for the house, all from Google 3D Warehouse ${ }^{\mathrm{TM}}$. The main furniture included models such as chairs, couches, beds, curtains, and kitchen and bathroom items (Figure 1).

The system was designed as a collaborative virtual environment (CVE), where two networked users (i.e. a participant and an experimenter) interact in one virtual environment (similar to the concept of multiplayer virtual games). This design allowed for remote communication and collaboration between the two users. The game engine Unity $3 \mathrm{D}^{\mathrm{TM}}$ was used to build the system. The CVE provided two interfaces/applications that used the same virtual world and were synchronised with each other (i.e. one view for the participant and one view for the experimenter). Each interface enabled specific functionalities required for its user's tasks. More details of the system schematic architecture are illustrated in the following diagram (Figure 2).

To build the networking component of the system, Unity Networking was used. The two Unity applications were connected via a Master Server. First, the participant's application starts and is registered with the Master Server. The experimenter then joins the already running connection.

The participant was represented in the virtual world using an avatar entity, which enabled her/him to move, navigate, interact, and respond within the environment. The interaction in the environment was designed and implemented in a direct way: to perform any interaction, the participant used a standard gaming joystick to pick up objects, hold them up, move with them, and then put them down by pressing the joystick button. No complex animated avatar was used for the participant. Figure 3 shows an example of a participant (first-person view) picking up a toy, holding it (appears in front the participant), moving with it, and finally placing it in a toy box.

To enable collision, a sphere/capsule collider was added to the avatar, which made it naturally collide with objects when moving in the scene rather than going through them (e.g. with walls and furniture).

The connection over the network detects the inputs of the participant and applies them to the avatar, and then sends them across the network to the experimenter application for synchronisation. The participant 

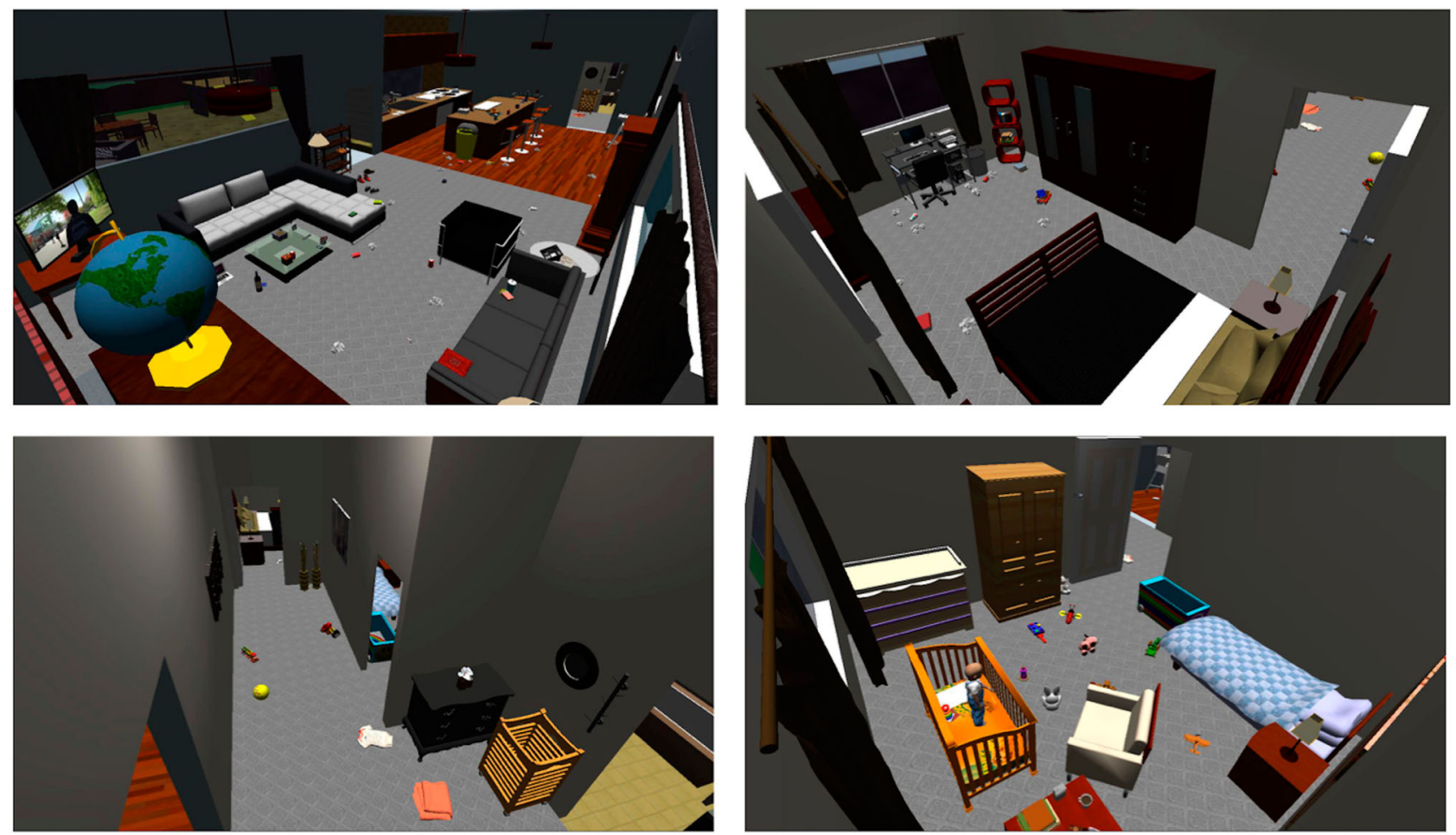

Figure 1. Virtual house test environment.

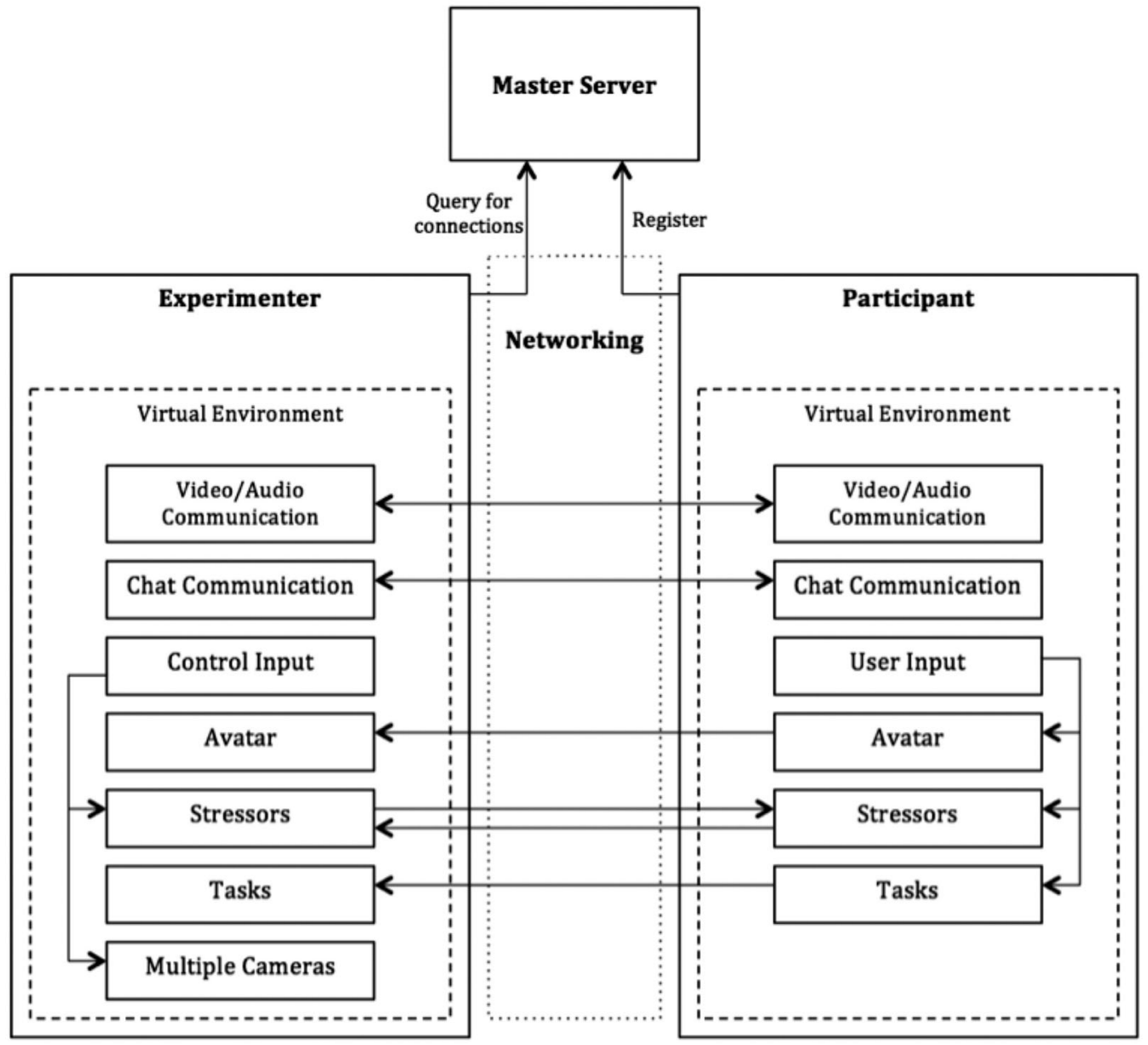

Figure 2. Schematic of the system architecture (including data streams). 

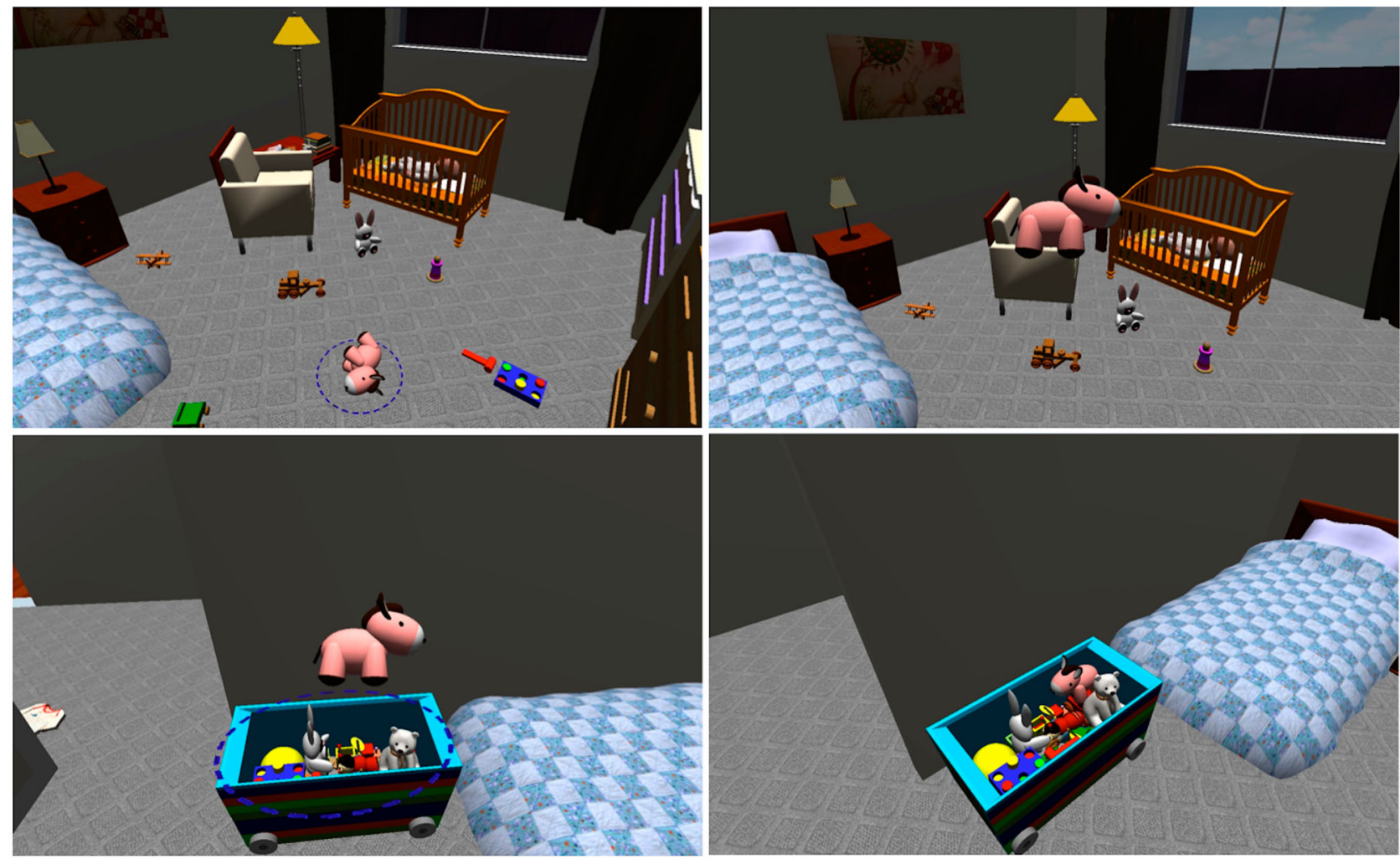

Figure 3. Example of an interaction in the environment.
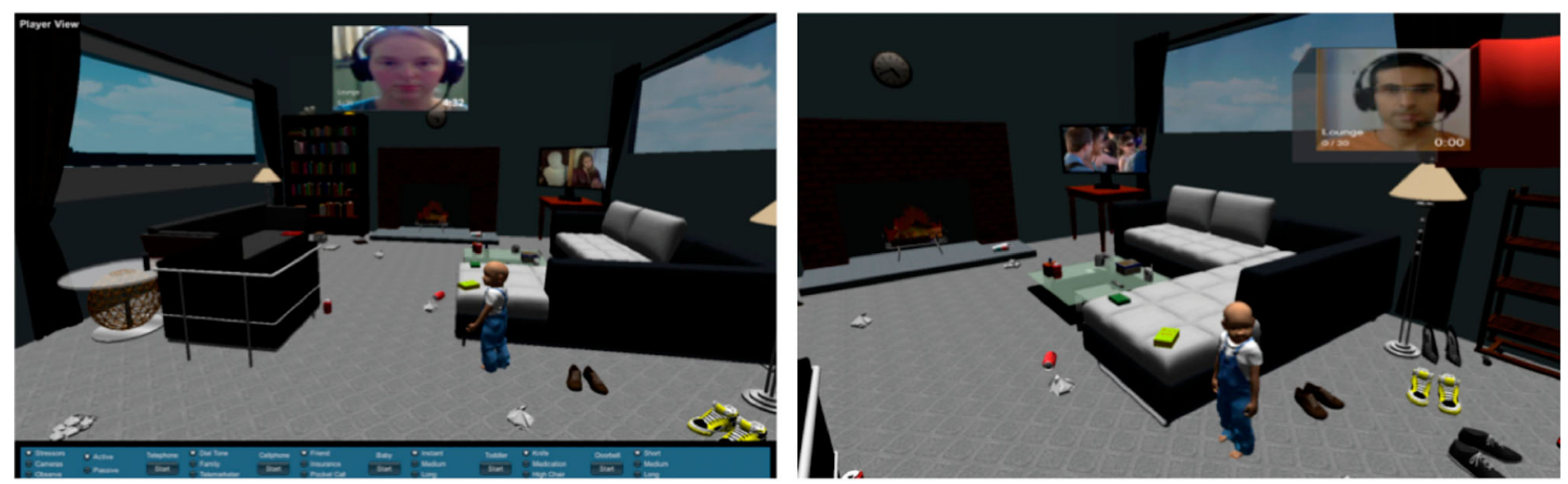

Figure 4. User interfaces: experimenter (left) and participant (right).

included the avatar's head movement and rotation and the object interaction. The experimenter interface provided more control over the system. When the application starts, the experimenter has a first-person view from the participant's perspective. This means the experimenter sees exactly what the participant sees (WYSIWIS). At the bottom of the interface, a control area is located for managing the virtual stressors (starting and stopping them) and changing the views inside the house. The experimenter actions applied to the stressors were synchronised to the participant application (this is the primary exposure process). The multiple views functionality of the experimenter allowed him/her to switch to a non-first-person view of the participant, then the simple avatar representation can be seen moving in the virtual world (additional observation functionality).

The system supported video communication to enable the two users to communicate in a shared environment. This function was implemented in the system using a videoconferencing communication library called Video Chat developed by Midnight Status (Status 2014). Using this library, the video and audio streams were processed and queued, then sent over the network 

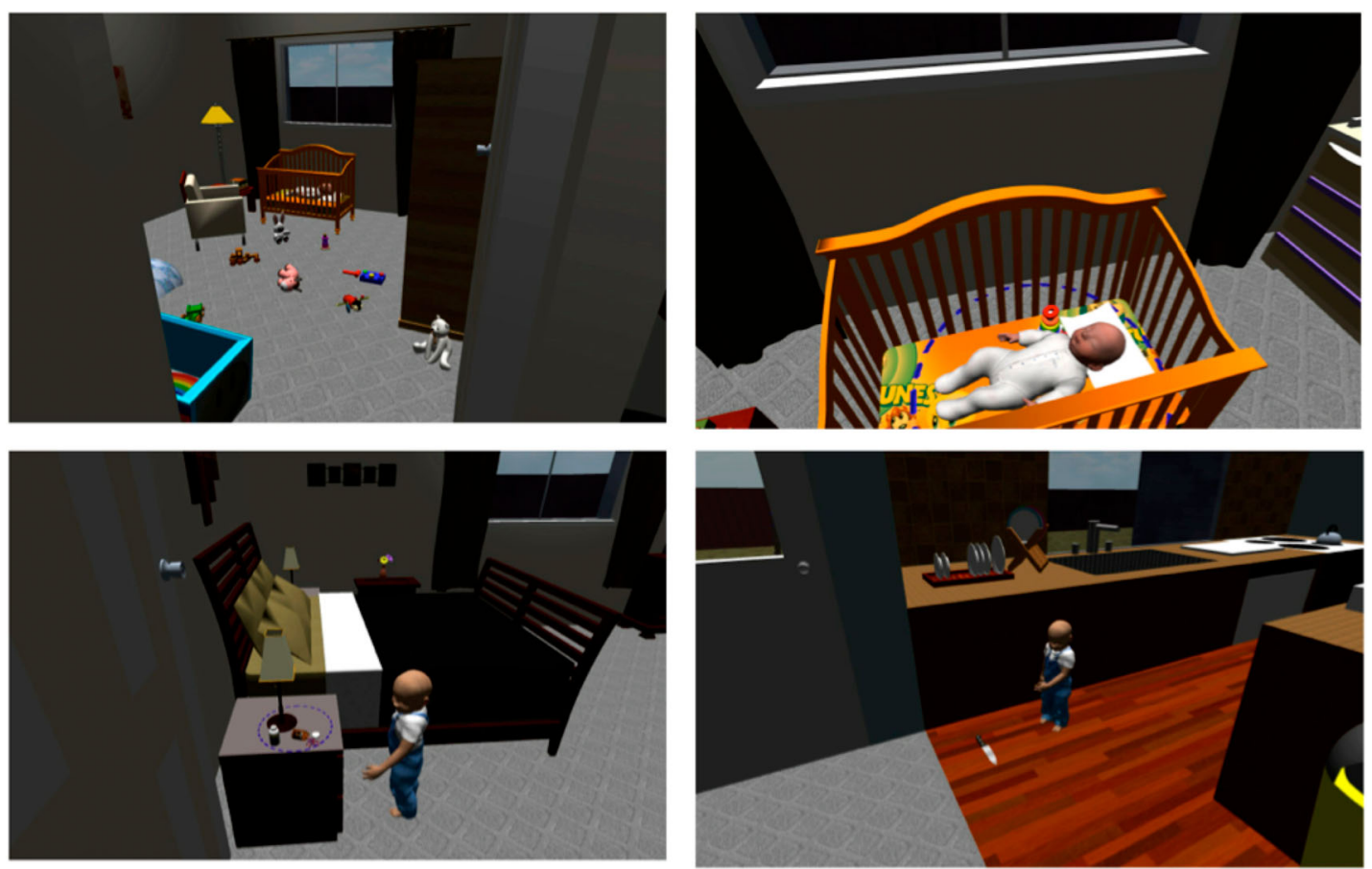

Figure 5. Examples of the VR stressors (clockwise from top left): a messy room to be cleaned up, a baby crying in the cradle, a toddler near medication, and a toddler near a knife.

in point-to-point streams between the two networked applications. A webcam image was compressed to $4 \mathrm{~K}$ pixels; this was done at 30 frames per second. Because the video communication screens in the system were small, the resolution was kept low, so there was no need for a high-resolution image. This also resulted in low required bandwidth. The screen size of the videoconferencing window for both users was the same, $85 \mathrm{~mm} \times$ $55 \mathrm{~mm}$ on monitor. The total bandwidth was about $270 \mathrm{kB}$ per second for the communication (video and audio).

Figure 4 shows screenshots of the graphical user interface for both the participant and the experimenter.

Based on the identified stressors listed earlier, a set of VR representations was developed in the virtual environment (Table A1, Appendix). All these virtual stressors focus on those that might typically arise for parents in a house environment after having a new child (examples in Figure 5).

\section{Method of the experiment}

This experiment investigated the influence of the developed VR stimuli on participants' levels of stress and anxiety. The resulting stress and anxiety were measured using three standardised psychological tests and two physiological measures (see method section 5.4). Stress and anxiety in this context are overlapping concepts, and henceforth will be referred to only as stress for simplicity. Exposure to the VR stressors lasted for seven minutes and could be present or absent depending on the session. During the experiment, the participants were asked about their stress, sense of presence, workload, and task completion.

Two main questions were examined:

- To what extent do the VR stimuli have an influence on levels of stress of the participant?

- Which relationships exist between levels of stress and sense of presence, workload, and task completion performance in the virtual environment?

\subsection{Experimental design and ethics}

The experiment followed a within-subjects design with three levels (baseline versus non-exposure versus stress exposure). Additionally, all participants performed two task conditions in the virtual environment. The main task was identical in both conditions; however, the Non-exposure Condition had no exposure to the stressors, whereas in the Exposure Condition, an exposure to the stressors was applied. To avoid any possible learning effect, the conditions were fully counterbalanced in a randomised order. The VR stressors in the Exposure Condition were also applied in a counterbalanced randomised order. This was to control the relationship between the stressors' orders and their influence. 
Using techniques designed to stress participants was the central ethical consideration. This experiment was approved by the University of Otago Ethics Committee (Reference: 15/021). The safety of the participants was taken seriously during the experiment to protect them from any possible physical and mental harm. The participants were provided with information sheets prior to the experiment, and a written consent form was signed and obtained from each participant.

\subsection{Participants}

A total of 54 adult participants (34 females and 20 males) took part in the experiment. The age of the participants ranged from 18 to 62 years $(M=28.67, \mathrm{SD}=9.85)$. The dominant ethnicity was New Zealand European by 31 participants, and the marital status varied (i.e. single: 21; married: 17; partnership: 12; divorced: 2; and other: 2). Overall, 34 participants did not have any children, while 20 participants reported having children (two female participants indicated that they have recently given birth, within the past six months).

Participants were recruited from the University of Otago by advertising around the university. The majority of them were university students; staff were also included. None of the participants had any prior knowledge of the experiment. All participants were given a \$20 gift voucher after completing the experiment as an appreciation for their participation.

\subsection{Apparatus}

The participant and the experimenter were located in the same room during the experiment. Two desks containing the hardware set-ups were appropriately arranged in one physical room, separated from each other through several cubical partition walls. This was to make the
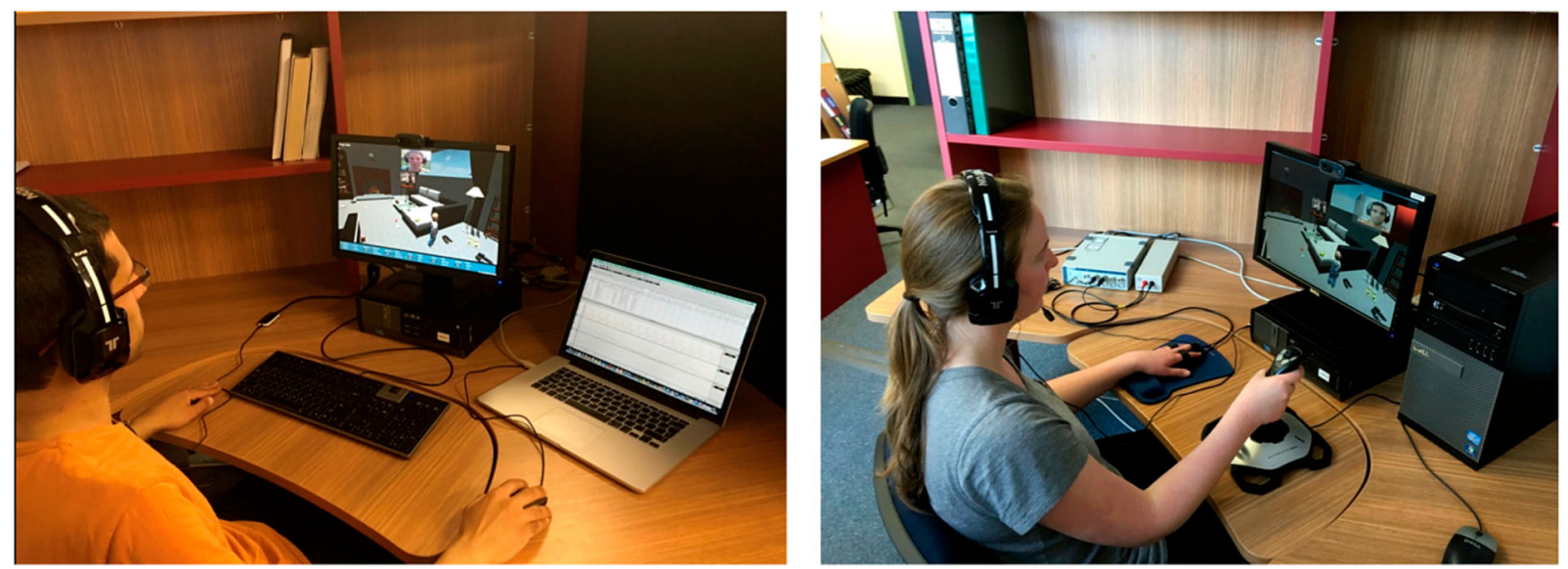

participant and the experimenter see and hear each other only via the system (Figure 6).

Almost identical hardware set-ups were used by the participant and the experimenter (Windows 7, Dell OptiPlex 3020 PC (Intel Core TM i5-4670@3.40 GHZ, RAM 8 GB), LCD screen (Dell E1910C, 19", $1440 \times$ 900), Logitech HD webcam C270, Tritton Kunai stereo headset). The experimenter operated the system with a standard computer mouse, while the participant used a standard gaming joystick (Logitech X3D).

In addition, the present experiment investigated the physiological reactions of the participants (HR and galvanic skin response (GSR)). To gather and record the participants' physiological data during the experiment, data acquisition devices/systems were used (ADInstruments). These instruments were a PowerLab system, Pulse Transducer, GSR Amp, and GSR Finger Electrode (Figure 7). The software LabChart was used to capture, visualise, and store the collected data from the instruments. The experimenter used an additional laptop dedicated only for capturing and managing the physiological data (MacBook Pro, 15-inch, 2.7 GHz Intel Core i7, 16 GB DDR3).

\subsection{Measures}

Data were gathered using four approaches: self-report questionnaires, task completion performance data (in the virtual environment), physiological measures, and semi-structured interviews.

\subsubsection{Self-report questionnaires}

Demographic information: several characteristics were gathered about the participants, including gender, age, marital status, whether the participant has children or experience looking after children, and the participant's level of expertise in playing VR games.

Figure 6. Hardware set-ups (experimenter left and participant right). 


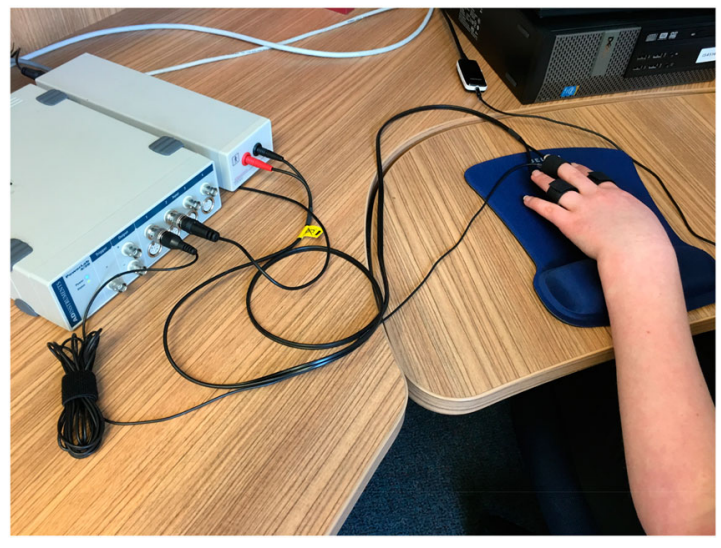

Figure 7. The devices attached to a participant during the experiment.

State-Trait Anxiety Inventory (STAI): STAI's Form ' $\mathrm{Y}$ ' was used to measure perceived anxiety (Spielberger and Gorsuch 1983). This measure consists of 40 questions using a 4-point Likert-like scale measuring two types of anxiety: state anxiety (items 1-20) and trait anxiety (items 21-40). The higher the item score, the higher the anxiety level. State or situational anxiety items were more appropriate and fit for the present experiment design. Thus, they were employed to measure the change in the anxiety level.

Subjective Units of Discomfort Scale (SUDS): a scale for measuring the subjective intensity of discomfort or anxiety experienced by an individual. A Likert-like scale was used, ranging from ' 0 totally relaxed' to ' 10 highest anxiety/discomfort that you have ever felt' (Wolpe 1958).

Subjective stress: a one-item question for subjective stress was used to directly ask how stressed participants were feeling (perceived stress). This one-question item used a Likert-like scale from ' 0 - not at all stressed' to ' 10 - extremely stressed'.

Workload (NASA-Task Load IndeX (TLX)): perceived workload in the VR environment was measured using the NASA-Task Load Index. This is a subjective multidimensional tool that was used to assess the task performance effectiveness. It measures six aspects related to task demands (i.e. mental demand, physical demand, and temporal demand) and the individual's reactions (i.e. performance, effort, and frustration) (Hart 2006). The original six items were used with modified Likert-like scales ranging from ' 0 very low' to ' 10 very high'. This minor modification to the scale was done due to the consistency with the other measures in the questionnaire.

Igroup Presence Questionnaire (IPQ): sense of presence in the VR environment was assessed using the IPQ designed by Schubert, Friedmann, and Regenbrecht (2001). It consists of 14 items on 7-point Likert-like scales ranging from -3 to 3 . The IPQ construct measures three important components related to presence: involvement (level of awareness of the VR environment), spatial presence (the connection between the VR environment and the real physical environment), and realism (the feeling of how real the VR environment is).

Additional measures for the VR stressors: to provide more assessment and in-depth evaluation about the VR stressors, additional measures were used to examine each stressor individually and in combination with the others. The participants were asked to rate each stressor based on levels of (1) awareness, (2) stress, (3) involvement, and (4) realism. At the end, the same questions were asked to examine these four factors when dealing with multiple or a combination of stressors. For scoring, Likert-like scales were used ranging from 0 (not at all) to 10 (very).

\subsubsection{Task completion performance}

This measured how successful the participant was in completing the main task in the VR environment in the given time (Section 5.5), which was measured by the number of objects collected successfully. Higher levels of completion mean higher task completion performance.

\subsubsection{Physiological data}

Changes in physiological data were measured as an additional examination for the elicited stress responses to the VR stressors.

The HR and GSR were the two types of physiological data that were gathered.

HR was monitored continuously using a pulse transducer. The transducer was applied around the participant's middle finger of the non-dominant hand and then connected directly to the PowerLab main unit.

GSR or skin conductivity is a change in the electrical properties of the skin. The GSR finger electrode was applied to the index and ring fingers of the participant's non-dominant hand and then connected to the GSR amplifier of the PowerLab unit.

\subsubsection{Semi-structured interviews}

The self-reporting questionnaire and physiological data often do not answer the Why questions. To explain and understand participants' perceptions and reactions, a semi-structured interview was conducted with each participant at the end of the experiment. Open-ended questions were asked covering the aspects of the VR environment, VR stressors, task, stress, and sense of presence. 


\subsection{Scenarios, tasks, and VR exposure}

\subsubsection{Non-exposure condition}

In a CVE displayed on screen, participants experienced and performed simulated everyday family activities designed to be similar to those in real life. In this scenario, the participant performed the role of a parent tidying up her/his messy house. This main task 'tidying up' was suitable in giving the experiment scenario. It was identified based on the results from the stressors identification process. Using the joystick, this task involved the act of moving around the messy house, picking up the task items, and then placing them in the appropriate places (taking the rubbish to the waste bin, the books to the bookshelf, the children toys to the toy box, the dirty clothes to the washing basket, etc.). The participant was encouraged to tidy up the house in seven minutes. The (remote) experimenter observed the participant's activity in the VR environment. When necessary, the experimenter could provide some help when asked by the participant. The video communication function facilitated the remote communication between the two parties.

\subsubsection{Exposure condition}

In this condition, the participant and the experimenter carried out the same scenarios and tasks from the nonexposure condition. The participant's main task was still tidying up the virtual house in the same approach and time frame. In addition, while the participant was doing the main task, the experimenter controlled (trigger and stop) the everyday family stressors in the virtual house. The participant interacted and responded to these stressors while carrying out the tidying activity. The word 'stressors' was not used in the instructions provided to the participants at all (i.e. instruction sheet). Instead, the word 'situations' was used as an indirect reference for the VR stressors. This was to avoid any possible pre-influence on how the participants would feel about them. The participants had no knowledge of what the situations were that they needed to deal with. All they knew was that as parents they had to tidy up their messy house and take care of any other situations that may happen in their house. The participants knew that they were parents of two children in the house, a newborn child and a two-year-old toddler (this information was provided in the instruction sheet). Also, they were informed that as parents they needed to keep a frequent eye on where their children were and what they were doing.

The task completion performance in the VR environment was measured based on how many tidying items were collected successfully in the seven minutes of the condition. The higher the number, the higher the task completion performance level.

To perform the exposure, the experimenter triggered each individual stressor separately; then, at the end of the condition, a combination of all stressors was triggered together. The rationale behind this was to be able to evaluate the effect of each stressor alone, then, the combination of the stressors, which is likely to have a greater impact. The exposure started after fifteen seconds from the beginning of the condition. Overall, each stressor was triggered for 30 seconds and a gap of nonexposure of 10 seconds was placed between the stressors. The timing for the toddler stressor was different due to the three situations. Each situation was triggered for 10 seconds, planned appropriately when the participant was going towards the area where the situation was.

\subsection{Procedure}

Data were only collected from participants and not from the experimenter. The experiment took approximately 45 minutes.

Before the experiment began, all participants were provided with information sheets and consent forms, which provided detailed explanations. If they agreed and signed the consent forms, they proceeded to the experiment.

The data collection for the self-report questionnaires was divided into three parts: before the first condition (baseline), after the first condition, and after the second condition. Physiological data were collected continuously only during the two conditions (HR and GSR). No baseline of the physiological data was measured. These self-report questionnaires and physiological data were needed to determine the influence of the changes in the virtual environment, mainly that of the VR stressors on the participants' feelings and perceptions while carrying out the tasks.

Before the first condition, the first set of questionnaires was given to the participant to complete (demographic, STAI, Subjective Stress, and SUDS). Then, the participant went through a short demonstration and practised how to operate the system (software and hardware). Once the participant became familiar with the equipment, an instruction sheet was provided that described the scenario and task step by step.

To ensure equal treatment for all participants, the experimenter followed a predesigned instruction sheet during the experiment. It explained the tasks for the experimenter in detail.

When the participant was ready for the first condition, the devices for collecting the physiological data were attached to the participant's hand (the pulse 
transducer for HR and the GSR finger electrode). The participants were informed to avoid any movement of the hand (including fingers) to avoid having noise in the data. They were given the time to choose a comfortable location for the hand before the start.

After finishing all essential preparation, the first condition started. During this condition, the participant and the experimenter performed their tasks as explained earlier. The physiological data were collected during the entire first condition of seven minutes. When the condition was completed, a second set of questionnaires was completed, which took approximately five minutes (STAI, Subjective Stress, SUDS, NASA-TLX, and IPQ).

Next, the second condition of another seven minutes started, which also contained a measurement of the physiological data. This condition included the exposure process that was explained earlier (the condition order was randomly balanced). When the second condition was completed, the collection of the physiological data ended and the devices were disconnected. Then, a third and last set of questions was completed by the participants (STAI, Subjective Stress, SUDS, NASA-TLX, IPQ, and the additional measures for the VR stressors).

The final stage of the data collection process was the semi-structured interviews. They were conducted at the end of the experiment, which focused on open-ended questions. Each interview took approximately $10 \mathrm{~min}-$ utes. After finishing the interviews, the participants were thanked and given gift vouchers.

\section{Results}

In this section, we report on the results from the selfreport questionnaires, task completion performance, physiological measures, and semi-structured interviews.

\subsection{Self-report questionnaires}

Distribution tests were performed for the measurement variables. Histogram outputs and a Shapiro-Wilk test showed that all the tested variables were normally distributed except for the task completion performance. Repeated measures ANOVA was used to test subjective stress, STAI, and SUDS.

\subsubsection{Subjective stress}

For subjective stress, Mauchly's test indicated that the assumption of sphericity had been violated, $\chi^{2}(2)=$ $32.18, p<.001$; therefore, degrees of freedom were corrected using Greenhouse-Geisser estimates of sphericity $(\varepsilon=.68)$. The results show that there was a significant change in subjective stress perceived by participants across the three times during the experiment, $F(1.36$,

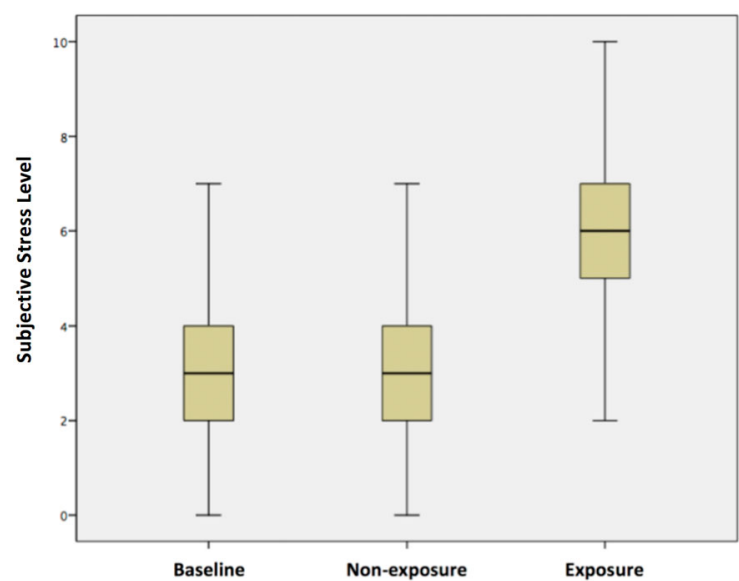

Figure 8. Means for the reported subjective stress.

$72.52)=81.60, p<.001, \omega 2=0.60$. Post hoc pairwise comparison with adjustment for multiple comparisons using Bonferroni method was used and showed that subjective stress was significantly higher in the exposure condition than the other two conditions (non-exposure and baseline). The participants were significantly more stressed in the exposure condition $(M=6.28, \mathrm{SD}=$ 1.91) compared to the non-exposure condition and baseline, respectively $(M=3.31, \mathrm{SD}=1.57$, means difference $=2.96, p<.001) ;(M=3.02, \mathrm{SD}=1.59$, means difference $=3.25, p<.001)$. The results suggested no significant difference in the scores between the non-exposure condition and baseline (means difference $=0.296, p=.209$ ). Figure 8 shows the difference in means of subjective stress during the experiment.

\subsubsection{State-trait anxiety - STAI}

When examining the STAI variable, Mauchly's test showed that the assumption of sphericity had been violated, $\chi^{2}(2)=86.75, p<.001$. Correction for degrees of freedom was employed using Greenhouse-Geisser estimates of sphericity $(\varepsilon=.55)$. Like subjective stress, the results indicated that there was a significant difference in STAI scoring during the experiment, $F(1.10,58.51)$ $=496.62, p<.001, \omega 2=0.90$ (Figure 9). Further analysis using post hoc pairwise comparison (with adjustment for multiple comparisons using Bonferroni method) reported that the STAI level was significantly higher in the exposure condition compared to the non-exposure and baseline conditions. Results showed that the participants were significantly more anxious when exposed to the VR stressors $(M=56.31, \mathrm{SD}=8.42)$ than during the non-exposure condition and baseline, respectively $(M=30.28, \quad \mathrm{SD}=5.91$, means difference $=26.03, \quad p$ $<.001 ; M=29.69, \mathrm{SD}=6.06$, means difference $=26.63$, $p<.001)$. No significant difference was found between 


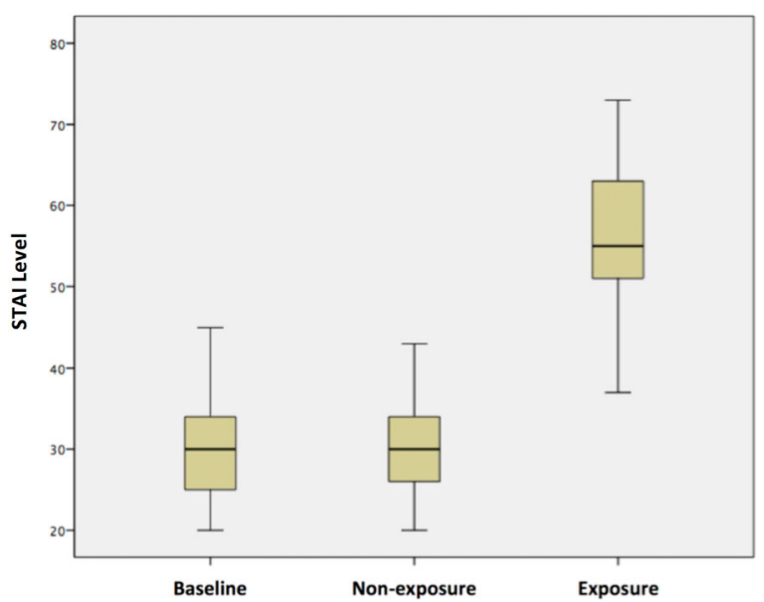

Figure 9. Means for the reported STAI.

the non-exposure condition and baseline (means difference $=0.59, p=.187$ ).

\subsubsection{Subjective of discomfort - SUDS}

The results of SUDS showed that the sphericity's assumption had been violated, $\chi^{2}(2)=43.92, p<.001$. Again, degrees of freedom were corrected using Greenhouse-Geisser estimates of sphericity $(\varepsilon=.63)$. Similarly to previous results, the results suggested that there was a significant change in SUDS scores, $F(1.27,67.50)=$ 205.79, $p<.001, \omega 2=0.79$ (Figure 10). Based on the post hoc pairwise comparison (with adjustment for multiple comparisons using Bonferroni method), SUDS scores were significantly higher in the exposure condition compared to the others. The SUDS level significantly increased during the exposure of the VR stressors $(M=6.91, \quad \mathrm{SD}=1.62) \quad$ compared to the condition with no stressors and baseline, respectively $(M=3.11, \mathrm{SD}=1.61$, means difference $=3.79, p<.001$; $M=2.91, \mathrm{SD}=1.58$, means difference $=4.00, p<.001$ ).

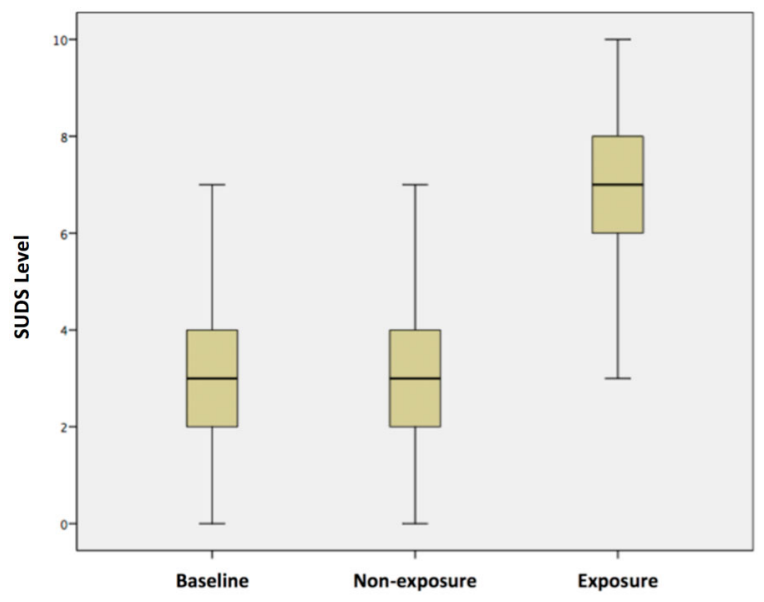

Figure 10. Means for the reported SUDS.
Analysis revealed that there was no significant difference between the condition with no stressors and baseline (means difference $=0.20, p=0.210$ ).

\subsubsection{Workload (NASA-TLX) and sense of presence (IPQ)}

Since the variables workload (NASA-TLX) and sense of presence (IPQ) consist of two conditions (non-exposure condition and exposure condition), two-tailed paired samples $t$ tests were used (Figure 11). Results revealed that there was a significant difference in the scores for NASA-TLX in the exposure condition $(M=6.10, \mathrm{SD}=$ 1.26) and the one with no exposure $(M=4.41, \mathrm{SD}=$ $1.53) ;(t(53)=9.25, p<.001)$. There was also a significant difference in IPQ, showing that the exposure condition $(M=1.42, \mathrm{SD}=0.44)$ received significantly higher scores compared to the non-exposure condition $(M=0.02$, SD $=1.02) ;(t(53)=11.51, p<.001)$.

A Pearson correlation analysis revealed a moderate positive correlation between NASA-TLX and IPQ, $r=$ $0.43, n=54, p=.001$ (Figure 12). Participants who reported higher workload indicated higher level of sense of presence. NASA-TLX also showed a moderate positive correlation with STAI, $r=0.32, n=54, p=.01$. No other significant correlations were found between NASA-TLX/IPQ and the other variables.

\subsubsection{Additional measure for the VR stressors}

The results showed that all participants were very aware of all the VR stressors in the environment, reporting an overall $M$ of 8.38 out of 10 . As expected when testing how stressful each stressor was, the results showed that the combination of all stressors together had the strongest impact $(M=8.22)$, followed by the crying baby $(M=7.43)$ and the toddler stressor $(M=6.65)$. The lowest was the pet noise $(M=3.69)$.

The involvement level was different between the active and the passive stressors. On the one hand, the participants devoted a reasonably high level of involvement with the active stressors (crying baby $M=8.76$, untidy/messy house $M=8.44$, doorbell $M=7.52$, telephone $M=7.43$, cell phone $M=7.02$, toddler $M=7$, and the combination $M=6.96$ ), whereas the passive stressors received a low level of involvement (neighbour's noise $M=1.24$, street noise $M=1.56$, and pet noise $M=2.20$ ).

The level of realism of all the VR stressors was reasonable and above midpoint $(M=6.11)$. The highest level was for the toddler $(M=7.11)$, followed by the combination of stressors $(M=7.02)$, and then the crying baby $(M=6.86)$. 

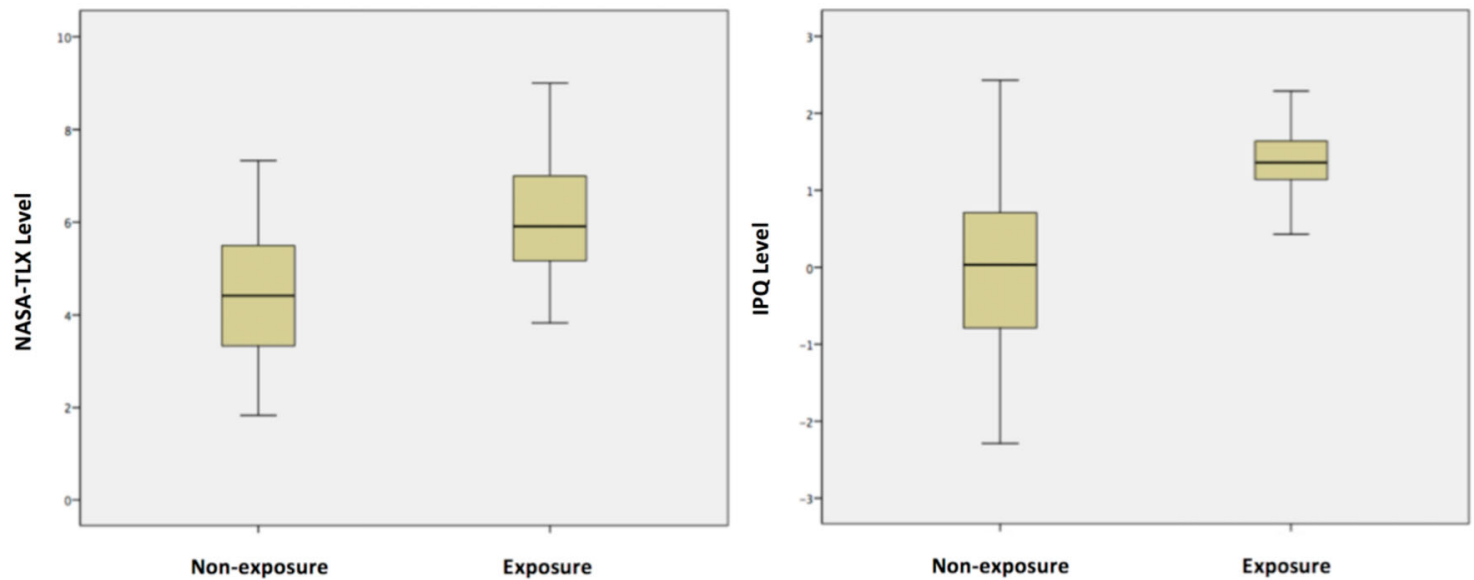

Figure 11. Means for workload as measured by the NASA-TLX (figure left) and the reported sense of presence IPQ (figure right).

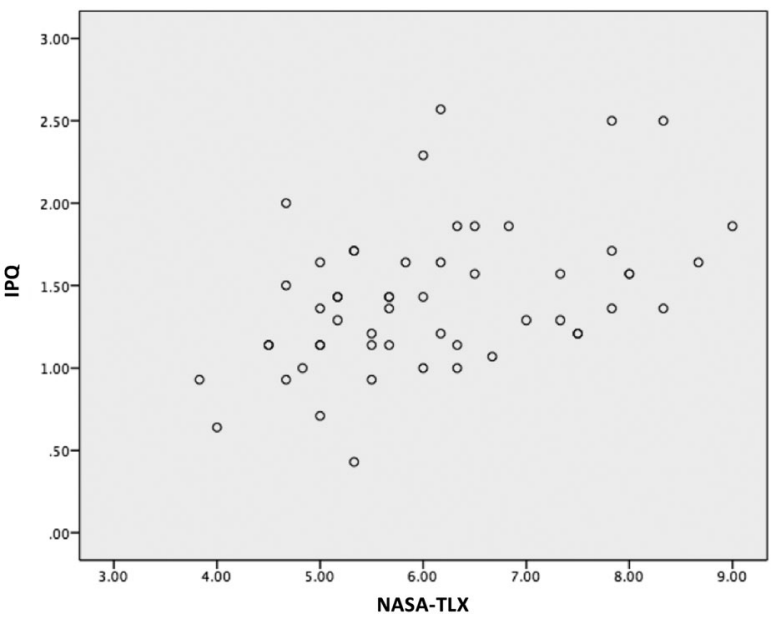

Figure 12. Scatter plot of correlation between workload (NASA$\mathrm{TLX}$ ) and sense of presence (IPQ).

\subsection{Task completion performance}

The task completion performance measured how successful the participant was in completing the main task (tidying up the house). This implies how many task items were collected successfully. Results showed that participants had lower task completion performance in the exposure condition $(M=30.50, \mathrm{SD}=10.30)$ than in the non-exposure one $(M=56.67, \mathrm{SD}=15.19)$, with mean difference of 26.16 .

\subsection{Confounding variables}

The results may be influenced by some characteristics of the participant. Two relevant confounding variables were examined during the data analysis: gender and whether the participant has children.

The analysis showed no significant effect of gender on any of the examined variables across all conditions.
Having children, on the other hand, had a significant effect on some of the variables. The three variables used to measure levels of stress (subjective stress, STAI, and SUDS) had been influenced by whether or not the participant was a parent. This significant impact only occurred during the exposure condition. Two-tailed independent samples $t$ tests showed that non-parent participants reported significantly higher scores for subjective stress, STAI, and SUDS, during the exposure condition compared to parent participants (Table 1 shows the results).

No significant effect was detected of parenting on the baseline or the non-exposure condition of these three variables. For NASA-TLX and IPQ, having children did not show any significant effect on scores in both conditions too.

\subsection{Physiological data results}

First, the gathered data were checked for noise that resulted from hand movement. Twenty-one participants were excluded due to noise in the data. The analyses of the physiological data were based on the data from the remaining 33 participants ( 21 females, 12 males). The age of the participants ranged from 18 to 50 years $(M$ $=29.18, \mathrm{SD}=9.59$ ).

Normality tests were then conducted on the variables for the two conditions. Results from the Shapiro-Wilk

Table 1. $t$ test results for the influence of parenting.

\begin{tabular}{llrrrr}
\hline Variable & \multicolumn{1}{c}{ Groups } & $M$ & SD & $t(52)$ & $P$ \\
\hline Subjective stress & Non-parents & 7.06 & 1.57 & 4.58 & $<.001$ \\
& Parents & 4.95 & 1.73 & & \\
STAI & Non-parents & 59.41 & 7.72 & 3.98 & $<.001$ \\
SUDS & Parents & 51.05 & 6.91 & & \\
& Non-parents & 7.50 & 1.33 & 3.93 & $<.001$ \\
& Parents & 5.90 & 1.61 & & \\
\hline
\end{tabular}



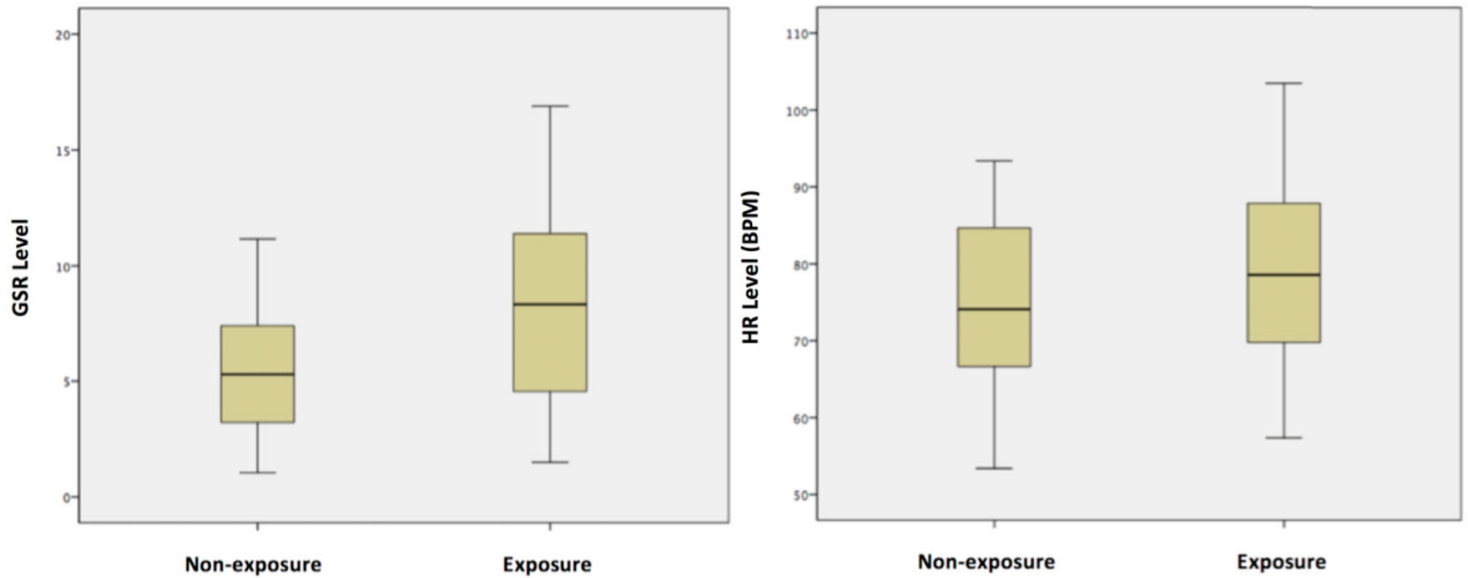

Figure 13. Means of physiological measures GSR (figure left) and HR (figure right).

test suggest that both GSR and HR data were normally distributed.

Outcomes showed that there were statistically significant differences of GSR and HR scores when comparing between the exposure condition and the non-exposure condition (Figure 13). A two-tailed paired samples $t$ test indicated that the GSR level was significantly higher in the exposure condition $(M=8.65, \mathrm{SD}=4.29)$ than in the non-exposure condition $(M=5.40, \mathrm{SD}=2.81) ; t$ $(32)=6.97, p<.001$. An average mean difference of $3.25 \mu$ Siemens was recorded between the two conditions. Furthermore, a two-tailed paired samples $t$ test showed that $\mathrm{HR}$ in the exposure condition $(M=79.39, \mathrm{SD}=$ 12.78) increased compared to that in the non-exposure condition $(M=74.75, \mathrm{SD}=11.60)$. The increase was statistically significant $t(32)=6.71, p<.001$. A 4.64 beats per minute was the average mean difference between the two conditions. No significant differences were found in the variances between the exposure and non-exposure conditions in both GSR and HR.

\subsection{Semi-structured interview results}

Four participants declined the interview, leaving 50 participants in the analysis. All interviews were audio recorded and transcribed. The transcripts were analysed using a GIA (Elo and Kyngäs 2008). This approach was conducted following the recommendations of Thomas (2006). The purpose was to identify emerging themes in the data. Text segments or sentences with similar meaning were gathered in several groups to create thematic categories. All categories were then organised into a hierarchal relationship based on the similarity in the meaning and topic, which allowed three major themes to emerge. Two coders assessed the coding consistency (inter-rater reliability) and member check (credibility). The inter-rater reliability was 93\% (raw agreement), and disagreements were discussed and resolved. Additionally, the member check resulted in points of view and minor modifications were discussed until agreement was reached and the interpretations of the findings were confirmed. As a result, 30 sub-categories were identified. The following sections show the main themes with summaries of sub-categories with quotes (the sub-categories were summarised given the length condition of this paper, without affecting the quality of the results).

\subsubsection{Theme 1: perceived stress in the virtual environment}

Participants provided statements that indicated their feeling of stress during the experiment. The findings showed a difference between the two conditions in terms of evoking stress. Three sub-themes emerged from this first theme:

(1) The majority of the participants $(96 \%, n=48)$ indicated that they were more stressed/anxious in the exposure condition compared to the non-exposure one:

the second session [the exposure condition] definitely was more stressful. (P1)

(2) Having determined that the exposure condition was perceived as more stressful, the participants were asked why they felt more stressed or anxious during this condition. They highlighted the following reasons as factors that created this level of stress in the virtual environment: too many things/tasks to do $(28 \%, n=14)$, too many things/tasks to do at once $(28 \%, n=14)$, looking after the children $(42 \%, n=21)$, mental demand $(16 \%, n=8)$, high levels of noise $(10 \%, n=5)$, unexpected events 
$(10 \%, n=5)$, and too many interruptions and distractions $(6 \%, n=3)$ :

'... because there are lots of things with high priority that got in the way of the original task' (P21) ... ' ... because lots of things happened at once and you don't have time to do them all' (P5)) ... ' '... when I saw the kid trying to eat the medicine I said oh my god my child is going to eat that medicine, I stressed even though it wasn't real' (P10) ... ' ... because there was too many demand and they are potentially urgent like the dangerous knife with the child and the baby crying, the sort of things you have to attend to and cannot wait' (P46) ... . '... cannot deal with screaming babies' (P28) .... '... because all the unexpected situations' (P3) ... . ... because you want to achieve the task and you have distractions especially toward the end' (P13)

(3) Differences and commonalities in parents and nonparents: some parents $(25 \%, n=13)$ were more familiar and close to the scenario. This was a result of the parenting scenario/tasks during the exposure condition, which are related to their real life:

... for me I felt I was familiar with all the situations in the second session [the exposure condition] because they happen often in my life as a mother. (P37, a mother)

Most of both parents and non-parents $(90 \%, n=45)$ paid more consideration towards the children compared to other stressors during the exposure:

... I was much more concerned about the toddler/baby than in accomplishing the tasks, they were my main focus ... I think in the back of my mind I was always worried where is the toddler was and what is getting into. (P37, a mother)

Some of the parents and some non-parents showed a common technique when dealing with the stressors $(20 \%, n=10)$. The technique reported was prioritising based on the importance of the task. From the findings, the children task was regarded as the most important task in almost all cases $(92 \%, n=46)$. This technique was described as a common approach that participants usually follow when facing a similar scenario in real life:

... you had to try to juggle many different things and I tried to prioritise with that as well ... I had to decide which one was more important like the baby and the toddler. (P11, non-parent)

\subsubsection{Theme 2: perceived sense of presence}

When the participants were asked about their feelings of presence in the virtual environment, they reported different perceptions during the experiment. Findings about sense of presence were revealed in three main subthemes: realism, spatial presence, and involvement:

(1) Most of the participants $(84 \%, n=42)$ reported that the virtual environment was more real in the exposure condition compared to the non-exposure one. Only few participants $(12 \%, n=6)$ felt that the non-exposure condition was more realistic due to the scenario in the environment, which was not similar to their real life:

I think the second session [the exposure one] was more realistic to me. (P1)

(2) The feeling of being physically present in the virtual environment varied during the experiment. The majority of the participants $(86 \%, n=43)$ stated that they felt greater spatial presence in the virtual environment during the exposure condition compared to the non-exposure one:

for me the one with the kids [the exposure condition] I felt more present. (P37)

(3) The participants described more involvement as another characteristic of the exposure condition. They found the environment more involving and engaging because of the higher level of interaction resulting from the stressors:

... also the interaction with everything happened, it was more interactive, you get more heavily involve. (P15)

\subsubsection{Theme 3: similarity and sensibility between the virtual environment and real life}

There was a clear connection between real-life experiences/scenarios and how participants perceived the virtual environment. Some participants $(40 \%, n=20)$ reported that they related more to the virtual environment during the exposure condition as it reflected a similar and sensible representation of the real life:

... this like in my real life, like when I have to tidy up I usually have to respond to extra stuff happening. (P15)

\section{Discussion}

The findings from this research obtained from healthy participants confirmed that virtual representations of typical everyday domestic stressors in a non-immersive VR environment can induce significant psychological and physiological reactions to stress. The examined stressors in this research were a type of mild stimuli that typically arise for parents in a house environment after having a newborn child. Unlike major stressors or 
significant life changes, these stressors tend to occur very often (Crnic and Greenberg 1990). In real life, such an individual event may not have strong effects on stress; however, it is the cumulative impact of a number of them over a period that makes them stressors (Crnic and Greenberg 1990). In the current VR environment/ scenario, participants felt significantly more stressed and anxious when confronted by these common stressors in the exposure condition compared to the non-exposure one. This was clearly indicated by the self-report measures (STAI, SUDS, and subjective stress). Similarly, changes were detected in participants' physiological responses during the experiment. Levels of HR and GSR showed a statistically significant increase in the exposure condition compared to the non-exposure one. This is supported by Meehan et al. (2003) reporting that HR and GSR tend to increase when exposed to stressful VR environments/situations. Furthermore, during the semi-structured interviews, almost all participants (96\%) confirmed this finding by clearly stating that they felt greater stress in the exposure condition resulting from the triggered stressors.

Stressors used in the experiment were analysed in order to better understand and explore their levels of influence on the participants. In the self-report questionnaire, participants subjectively indicated their perceptions of each stressor in terms of awareness, involvement, realism, and how stressful it was. The previous results section showed that all participants were very aware of all stressors in the environment, even if it was just presented on a desktop monitor screen, especially when the stimuli occurred together. The involvement level was high with all the active stressors (e.g. the crying baby, looking after the toddler, and the phones), as well as with the combination of the stressors and performing the main task (tidying up the house). However, there was low involvement with the passive stressors (e.g. neighbours' loud argument, noisy street sirens, and noisy dogs barking). This result was expected, as the active stressors required responses, while the passive ones did not. Low involvement did not necessarily reflect on low induced stress as shown in the results. All the stressors contributed to the increase in stress. Looking after the two children appeared to evoke more stress than the others when dealing with the stressors individually. The findings in the interviews support this quantitative result. About $42 \%$ of the participants stated that they perceived the task of looking after the two children as stressful. This can be explained by the apparent attention and consideration that were given to the children in the virtual environment. It seemed that the virtual children avatars were very effective in targeting participants' emotions similar to real-life situations, even if they were not highly photo-realistic. In particular, most of the parents linked this attachment to caring for their own children. During the exposure, a significant number of participants showed strong emotional attachment to the two children.

In addition, when responding to the multiple stressors at the same time, participants adopted a technique/solution from real-life experience, which also gave more focus to the children. The majority of parents and some non-parents followed a prioritising technique, dealing and responding to the most important and urgent event/stressor first. The children task was regarded as the most important task in almost all cases. This technique was described as a common approach that participants usually follow when facing a similar scenario in real life.

Nevertheless, it was the combination of stressors that had the strongest effect compared to any other individual stressor. This corresponds with real-life situations as it is shown that a combination of stressors would be likely to lead to greater impact compared to facing a single stressor (Bartenwerfer, 1960, 1963, 1969; Myrtek and Spital 1986). The factor 'Too many things/tasks to do at once' is also known to be stressful in real life (Kohn, Lafreniere, and Gurevich 1990; Kohn and Macdonald 1992). Participants strongly supported and confirmed this quantitative result during the interviews. In most feedback, participants indicated that responding to multiple situations at once was a difficult and stressful/anxious task during the exposure condition.

During the semi-structured interviews, more details were uncovered regarding the stressors. Participants stated in detail factors that made them feel more stressed and anxious in the environment, all of which were also reported to be everyday stressors in real-life situations. Also, these resulting factors did match some of those identified earlier during the three-stage process in the research, which focused on gathering and evaluating the implemented everyday stressors in the VR environment. The factors from the semi-structured interviews included the following:

- Too many things/tasks to do (Kanner et al. 1981; Holahan, Holahan, and Belk 1984; Bolger et al. 1989; Chamberlain and Zika 1990).

- Mental demand (Bolger et al. 1989).

- High levels of noise (Kanner et al. 1981; Holahan, Holahan, and Belk 1984; Kohn and Macdonald 1992).

- Unexpected events (Kanner et al. 1981).

- Too many interruptions and distractions (Kanner et al. 1981; Chamberlain and Zika 1990; Crnic and Greenberg 1990). 
The quantitative results revealed that gender of participants had no impact on their levels of stress during the whole experiment. Interestingly, the difference in whether or not the participant has children (parenting) was shown to have a significant effect on levels of stress. Only during the exposure condition did non-parents feel significantly more stressed and anxious compared to parents. This result suggests that to some extent nonparents and parents perceived the stressors in the VR environment differently. The findings from the interviews seem to provide some explanations for why this significant difference occurred. Focusing on the parenting scenario/tasks during the exposure condition, the majority of parents stated that they were very familiar with such an environment and scenario. As they are parents, they relate to similar circumstances in their real lives. In contrast, some non-parents indicated that they were not familiar with the parenting scenario and the tasks as they differ from what they interact and deal with in their real lives.

According to Schuemie et al. (2000), it is very important that the details in the VR environment match reality. It seems that participants' emotions and feelings in VR may differ from one to another based on their real-life experience. In the current finding, the familiarity with the scenario as well as the prior experience of dealing with similar real-life situations may have given parents a degree of resilience capability towards such an environment. Consequently, they felt significantly less stressed and anxious than non-parents who lack the familiarity and the prior experience in such situations. Some researchers have argued that repeated stress exposure can desensitise people to stimuli that may possibly create negative psychological reactions (Wiederhold, Bullinger, and Wiederhold 2006).

The level of the sense of presence was found to be significantly higher during the stressful condition compared to the natural one (non-exposure). As discussed during the review of the related work, it has been shown that sense of presence and emotions are likely to associate with each other in virtual environments (Bouchard et al. 2008; Pallavicini et al. 2013), in particular for immersive environments. Higher levels of emotions, such as stress, anxiety, or fear, can lead to a higher level of sense of presence. This connection was investigated in different scenarios such as fear of heights (Regenbrecht, Schubert, and Friedmann 1998; Schuemie et al. 2000; Michaud et al. 2004) and fear of snakes (Bouchard et al. 2008). Other studies demonstrated that a low level of sense of presence can reduce emotional reactions such as anxiety in VR environments (Pallavicini et al. 2013). In the current research, even though presence was significantly higher in the stressful/anxious environment, surprisingly, no significant correlations were found between presence and stress (i.e. subjective stress, STAI, and SUDS). Only a moderate positive correlation occurred between presence and perceived workload (NASA-TLX, a tool that measures task demands, i.e. mental demand, physical demand, and temporal demand) and the individual's reactions (i.e. performance, effort, and frustration) (Hart 2006). This means that participants who expressed the tasks as higher workload tended to feel a higher level of sense of presence in the environment, bearing in mind that perceived workload was significantly higher in the exposure condition as a result of the triggered stressors

From the interview results, participants explained that the significant increase in levels of presence in the exposure condition resulted from the higher levels of realism, spatial presence, and involvement, which are the main components of the sense of presence (Schubert, Friedmann, and Regenbrecht 2001). In fact, the increase in these three factors resulted from the triggered stressors. Participants clarified that the stressors created several elements in the environment, which in return boosted these three components. They included sound/ audio, more interactivity, focus and attention, and similarity and sensibility to reality.

While we found that this non-immersive system elicited actual stress responses, it would be worthwhile to investigate whether a fully immersive system, for example, with a HMD), would lead to even higher responses. The bespoken literature would suggest such an assumption.

\section{Conclusions and future work}

It is well established that especially immersive VR environments and stimuli related to significant life changes or scenarios can create emotional reactions such as stress, anxiety, or fear. However, no research has investigated whether non-immersive VR stimuli of common, mild stressors can produce similar outcomes. The main finding of the current research filled this gap by showing that desktop-monitor VR representations of common stimuli can in fact induce significant psychological reactions to stress. A mixed methodology approach was used in this research: quantitative (subjective self-reported measures), qualitative (semi-structured interviews), and physiological measures (HR and GSR). However, the quality of the physiological data is probably not valid enough to draw any serious conclusions mainly for three reasons: (1) there is too much noise in the data, mostly attributable to participants' hand movements and (2) we only considered averaged measures per session, which is a too coarse granularity. Proper marked time- 
series analyses would be required here. (3) Apart from measuring GSR and HR in the non-exposure condition, there was no actual baseline measurement. This should be added in future studies.

The sense of presence increased significantly during the stressful and anxious environment compared to the natural one. Higher levels of realism, spatial presence, and involvement were the driving factors for the overall increase in presence, which resulted from the triggered stressors. Hence, the stimuli presented leveraged the sense of presence, thus making the environment more a VR, again with a non-immersive VR system.

This research also showed that people's emotions and behaviours in VR environments might be influenced by their real-life experience. For example, in the current stressful environment, having prior experience and familiarity with a parenting environment appeared to make parents significantly less stressed and anxious compared to non-parents who lack such experience. Moreover, many participants showed a clear emotional attachment towards the two virtual children in the environment. They connected this strong reaction to their real-life situations where they usually prioritise and put children first.

Based on the overall findings of this research, results could possibly be generalised to other similar scenarios with different environments and/or to other types of everyday stressors using similar VR implementations.

In addition, future research should examine if the increase in stress is similar to the one produced by a real-life situations with similar characteristics.

The current VR implementation might offer future benefits in the area of stress desensitisation or enhancing people's resilience for such stressful environments in reality. We are currently working on this notion and continuing the research to expand its applications and psychological benefits.

In summary, we would like to emphasise that (1) mild stress stimuli can lead to actually perceived psychological responses, and (2) even non-immersive VR can be used to achieve this.

\section{Acknowledgements}

The authors wish to thank all participants who volunteered to take part in the experiment. Special thanks to Elgar Storm and Abdulaziz Alshaer for their help and support during this research. Also, we would like to thank Tim Jowett for his assistance during the data analysis procedure.

\section{Disclosure statement}

No potential conflict of interest was reported by the authors.

\section{References}

Alghamdi, M., H. Regenbrecht, S. Hoermann, T. Langlotz, and C. Aldridge. 2016. "Social Presence and Mode of Videocommunication in a collaborative Virtual Environment." In Proceedings of the 20th Pacific Asia Conference on Information Systems (PACIS 2016, June 27 July 1, 2016), Chiayi, Taiwan: AISeL.

Baños, R. M., C. Botella, M. Alcañiz, V. Liaño, B. Guerrero, and B. Rey. 2004. "Immersion and Emotion: Their Impact on the Sense of Presence." CyberPsychology \& Behavior 7 (6): 734 741.

Barfield, W., D. Zeltzer, T. Sheridan, and M. Slater. 1995. "Presence and Performance within Virtual Environments." In Virtual Environments and Advanced Interface Design, edited by Barfield Woodrow and Thomas A. Furness III, 473513. New York: Oxford University Press.

Bartenwerfer, H. 1960. "Herzrhythmik-Merkmale als Indikatoren psychischer Anspannung.” Psychological Beitrage 4 (7): 7-25.

Bartenwerfer, H. 1963. "Über Art und Bedeutung der Beziehung zwischen Pulsfrequenz und skalierter psychischer Anspannung." Zeitschrift für experimentelle und angewandte Psychologie 10: 455-470.

Bartenwerfer, H. 1969. "Einige praktische Konsequenzen aus der Aktivierungstheorie." Zeitschrift für experimentelle und angewandte Psychologie 16: 195-222.

Bolger, N., A. DeLongis, R. C. Kessler, and E. A. Schilling. 1989. "Effects of Daily Stress on Negative Mood." Journal of Personality and Social Psychology 57 (5): 808-818.

Botella, C., R. Banos, V. Guillén, C. Perpiñá, M. Alcaniz, and A. Pons. 2000. "Telepsychology: Public Speaking Fear Treatment on the Internet." CyberPsychology \& Behavior 3 (6): 959-968.

Botella, C., M. Juan, R. M. Baños, M. Alcañiz, V. Guillén, and B. Rey. 2005. "Mixing Realities? An Application of Augmented Reality for the Treatment of Cockroach Phobia." Cyberpsychology \& Behavior 8 (2): 162-171.

Bouchard, S., J. St-Jacques, G. Robillard, and P. Renaud. 2008. "Anxiety Increases the Feeling of Presence in Virtual Reality.” Presence: Teleoperators and Virtual Environments 17 (4): 376-391.

Chamberlain, K., and S. Zika. 1990. "The Minor Events Approach to Stress: Support for the Use of Daily Hassles." British Journal of Psychology 81 (Pt 4): 469-481.

Cooper, A., R. Reimann, and D. Cronin. 2007. About Face 3: The Essentials of Interaction Design. Indianapolis, IN: John Wiley \& Sons.

Crnic, K. A., and C. L. Booth. 1991. "Mothers' and Fathers' Perceptions of Daily Hassles of Parenting Across Early Childhood." Journal of Marriage and the Family 53 (4): 1042-1050.

Crnic, K. A., and M. T. Greenberg. 1990. "Minor Parenting Stresses with Young Children." Child Development 61 (5): 1628-1637. doi:10.1111/j.1467-8624.1990.tb02889.x.

DeLongis, A., J. C. Coyne, G. Dakof, S. Folkman, and R. S. Lazarus. 1982. "Relationship of Daily Hassles, Uplifts, and Major Life Events to Health Status." Health Psychology 1 (2): 119-136.

Diemer, J., G. W. Alpers, H. M. Peperkorn, Y. Shiban, and A. Mühlberger. 2015. "The Impact of Perception and Presence on Emotional Reactions: A Review of Research in Virtual Reality.” Frontiers in Psychology 6 (26): 1-9. 
Diemer, J., A. Mühlberger, P. Pauli, and P. Zwanzger. 2014. "Virtual Reality Exposure in Anxiety Disorders: Impact on Psychophysiological Reactivity." The World Journal of Biological Psychiatry 15 (6): 427-442.

Difede, J., and H. G. Hoffman. 2002. "Virtual Reality Exposure Therapy for World Trade Center Post-Traumatic Stress Disorder: A Case Report." Cyberpsychology \& Behavior 5 (6): 529-535

Elo, S., and H. Kyngäs. 2008. "The Qualitative Content Analysis Process." Journal of Advanced Nursing 62 (1): 107-115. doi:10.1111/j.1365-2648.2007.04569.x.

Garau, M., M. Slater, D.-P. Pertaub, and S. Razzaque. 2005. "The Responses of People to Virtual Humans in an Immersive Virtual Environment." Presence: Teleoperators and Virtual Environments 14 (1): 104-116.

Garcia-Palacios, A., H. Hoffman, A. Carlin, T. U. Furness, and C. Botella. 2002. "Virtual Reality in the Treatment of Spider Phobia: A Controlled Study." Behaviour Research and Therapy 40 (9): 983-993.

González-Franco, M., T. C. Peck, A. Rodríguez-Fornells, and M. Slater. 2014. "A Threat to a Virtual Hand Elicits Motor Cortex Activation." Experimental Brain Research 232 (3): 875-887.

Hart, S. G. 2006. "NASA-Task Load Index (NASA-TLX); 20 Years Later." In Proceedings of the Human Factors and Ergonomics Society Annual Meeting, (Vol. 50, No. 9, 904908). Los Angeles, CA: Sage Publications.

Hartanto, D., I. L. Kampmann, N. Morina, P. G. M. Emmelkamp, M. A. Neerincx, and W.-P. Brinkman. 2014. "Controlling Social Stress in Virtual Reality Environments." PLoS ONE 9 (3): e92804. doi:10.1371/journal.pone.0092804.

Heeter, C. 1992. "Being There: The Subjective Experience of Presence." Presence: Teleoperators and Virtual Environments 1 (2): 262-271.

Holahan, C. K., C. J. Holahan, and S. S. Belk. 1984. "Adjustment in Aging: The Roles of Life Stress, Hassles, and Self-Efficacy." Health Psychology 3 (4): 315-328. doi:10.1037/0278-6133.3.4.315.

Holmes, T. H., and R. H. Rahe. 1967. "The Social Readjustment Rating Scale.” Journal of Psychosomatic Research 11 (2): 213-218. doi:10.1016/0022-3999(67)90010-4.

Kanner, A. D., J. C. Coyne, C. Schaefer, and R. S. Lazarus. 1981. "Comparison of Two Modes of Stress Measurement: Daily Hassles and Uplifts Versus Major Life Events." Journal of Behavioral Medicine 4 (1): 1-39.

Klinger, E., S. Bouchard, P. Légeron, S. Roy, F. Lauer, I. Chemin, and P. Nugues. 2005. "Virtual Reality Therapy Versus Cognitive Behavior Therapy for Social Phobia: A Preliminary Controlled Study." Cyberpsychology \& Behavior 8 (1): 76-88.

Kohn, P., K. Lafreniere, and M. Gurevich. 1990. “The Inventory of College Students' Recent Life Experiences: A Decontaminated Hassles Scale for a Special Population.” Journal of Behavioral Medicine 13 (6): 619-630. doi:10. 1007/BF00844738.

Kohn, P., and J. Macdonald. 1992. "The Survey of Recent Life Experiences: A Decontaminated Hassles Scale for Adults." Journal of Behavioral Medicine 15 (2): 221-236. doi:10. 1007/BF00848327.

Krijn, M., P. M. Emmelkamp, R. Biemond, C. D. W. de Ligny, M. J. Schuemie, and C. A. van der Mast. 2004. "Treatment of Acrophobia in Virtual Reality: The Role of Immersion and Presence." Behaviour Research and Therapy 42 (2): 229-239.
Krijn, M., P. M. G. Emmelkamp, R. P. Olafsson, and R. Biemond. 2004. "Virtual Reality Exposure Therapy of Anxiety Disorders: A Review." Clinical Psychology Review 24 (3): 259-281. doi:10.1016/j.cpr.2004.04.001.

Loomis, J. M. 1992. "Presence and Distal Attribution: Phenomenology, Determinants, and Assessment." SPIE, Human Vision, Visual Processing, and Digital Display III 1666: 590-594.

McCall, C., L. K. Hildebrandt, B. Bornemann, and T. Singer. 2015. "Physiophenomenology in Retrospect: Memory Reliably Reflects Physiological Arousal During a Prior Threatening Experience." Consciousness and Cognition 38: 60-70. doi:10.1016/j.concog.2015.09.011.

Meehan, M., B. Insko, M. Whitton, and F. P. Brooks Jr. 2002. "Physiological Measures of Presence in Stressful Virtual Environments." ACM Transactions on Graphics 21 (3): 645-652.

Meehan, M., S. Razzaque, M. C. Whitton, J. Brooks, and P. Frederick. 2003. "Effect of Latency on Presence in Stressful Virtual Environments." In IEEE Virtual Reality, 2003. Proceedings, 141-148. Los Angeles, CA: IEEE.

Michaud, M., S. Bouchard, S. Dumoulin, X. Zhong, and P. Renaud. 2004. "Manipulating Presence and its Impact on Anxiety.” Cyberpsychology \& Behavior 7 (3): 297-298.

Mühlberger, A., H. H. Bülthoff, G. Wiedemann, and P. Pauli. 2007. "Virtual Reality for the Psychophysiological Assessment of Phobic Fear: Responses during Virtual Tunnel Driving." Psychological Assessment 19 (3): 340-346. Myrtek, M., and S. Spital. 1986. "Psychophysiological Response Patterns to Single, Double, and Triple Stressors." Psychophysiology 23 (6): 663-671.

North, M. M., S. M. North, and J. R. Coble. 1998. "Virtual Reality Therapy: An Effective Treatment for the Fear of Public Speaking." International Journal of Virtual Reality 3 (2): 2-6.

Pallavicini, F., P. Cipresso, S. Raspelli, A. Grassi, S. Serino, C. Vigna, S. Triberti, M. Villamira, A. Gaggioli, and G. Riva. 2013. "Is Virtual Reality Always an Effective Stressors for Exposure Treatments? Some Insights from a Controlled Trial." BMC Psychiatry 13 (1): 429-462.

Patterson, G. R. 1983. "Stress: A Change Agent for Family Process." In Stress, Coping, and Development in Children, edited by Norman Garmezy and Michael Rutter, 235-264. NewYork: McGraw-Hill.

Pertaub, D., M. Slater, and C. Barker. 2001. “An Experiment on Fear of Public Speaking in Virtual Reality." Studies in Health Technology and Informatics 81: 372-378.

Pertaub, D.-P., M. Slater, and C. Barker. 2002. “An Experiment on Public Speaking Anxiety in Response to Three Different Types of Virtual Audience." Presence: Teleoperators and Virtual Environments 11 (1): 68-78.

Regenbrecht, H. 2000. "Faktoren fuer Praesenz in virtueller Architektur" [Factors for a Sense of Presence in Virtual Architecture]. Doctoral thesis, Bauhaus University Weimar. Regenbrecht, H. T., T. W. Schubert, and F. Friedmann. 1998. "Measuring the Sense of Presence and its Relations to Fear of Heights in Virtual Environments." International Journal of Human-Computer Interaction 10 (3): 233-249.

Rizzo, A., J. Difede, B. O. Rothbaum, G. Reger, J. Spitalnick, J. Cukor, and R. Mclay. 2010. "Development and Early Evaluation of the Virtual Iraq/Afghanistan Exposure Therapy System for Combat-Related PTSD." Annals of the New York Academy of Sciences 1208 (1): 114-125. 
Rizzo, A., A. Hartholt, M. Grimani, A. Leeds, and M. Liewer. 2014. "Virtual Reality Exposure Therapy for CombatRelated Posttraumatic Stress Disorder." IEEE Computer 47 (7): 31-37. doi:10.1109/MC.2014.199.

Robillard, G., S. Bouchard, T. Fournier, and P. Renaud. 2003. "Anxiety and Presence During VR Immersion: A Comparative Study of the Reactions of Phobic and NonPhobic Participants in Therapeutic Virtual Environments Derived from Computer Games." CyberPsychology \& Behavior 6 (5): 467-476.

Rothbaum, B. O., L. Hodges, B. A. Watson, G. D. Kessler, and D. Opdyke. 1996. "Virtual Reality Exposure Therapy in the Treatment of Fear of Flying: A Case Report." Behaviour Research and Therapy 34 (5): 477-481.

Schubert, T., F. Friedmann, and H. Regenbrecht. 2001. "The Experience of Presence: Factor Analytic Insights." Presence: Teleoperators and Virtual Environments 10 (3): 266-281.

Schuemie, M., M. Bruynzeel, L. Drost, M. Brinckman, G. De Haan, P. Emmelkamp, and C. Van der Mast. 2000. "Treatment of Acrophobia in Virtual Reality: A Pilot Study." In Conference Proceedings Euromedia, 271-275. Antwerp, Belgium.

Seinfeld, S., I. Bergstrom, A. Pomes, J. Arroyo-Palacios, F. Vico, M. Slater, and M. V. Sanchez-Vives. 2016. "Influence of Music on Anxiety Induced by Fear of Heights in Virtual Reality." Frontiers in Psychology 6: 882-810.

Sheridan, T. 1992. "Musings on Telepresence and Virtual Presence." Presence: Teleoperators and Virtual Environments 1 (1): 120-126. doi:citeulike-article-id:3643828.

Slater, M. 2009. "Place Illusion and Plausibility Can Lead to Realistic Behaviour in Immersive Virtual Environments." Philosophical Transactions of the Royal Society B: Biological Sciences 364 (1535): 3549-3557. doi:10.1098/rstb.2009.0138.

Slater, M., C. Guger, G. Edlinger, R. Leeb, G. Pfurtscheller, A. Antley, M. Garau, A. Brogni, and D. Friedman. 2006. "Analysis of Physiological Responses to a Social Situation in an Immersive Virtual Environment." Presence: Teleoperators and Virtual Environments 15 (5): 553-569.

Slater, M., D. Pertaub, and A. Steed. 1999. "Public Speaking in Virtual Reality: Facing an Audience of Avatars." IEEE Computer Graphics and Applications 19 (2): 6-9.

Slater, M., and S. Wilbur. 1997. "A Framework for Immersive Virtual Environments (FIVE): Speculations on the Role of Presence in Virtual Environments." Presence: Teleoperators and Virtual Environments 6 (6): 603-616.

Spielberger, C. D., and R. L. Gorsuch. 1983. State-Trait Anxiety Inventory for Adults: Sampler Set: Manual, Tekst Booklet and Scoring Key. Palo Alto, CA: Consulting Psychologists Press.

Stetz, M., R. Wildzunas, B. Wiederhold, T. Stetz, and M. Hunt. 2006. "The Usefulness of Virtual Reality Stress Inoculation Training for Military Medical Females: A Pilot Study." Annual Review of Cyber Therapy and Telemedicine 4: 51-58.

Thomas, D. R. 2006. "A General Inductive Approach for Analyzing Qualitative Evaluation Data.” American Journal of Evaluation 27 (2): 237-246.

Video Chat 1.0091[Computer software]. 2014. Midnight Status. https://www.assetstore.unity3d.com/en/\#!/content/7447.

Walshe, D., E. Lewis, K. O'Sullivan, and S. I. Kim. 2005. "Virtually Driving: Are the Driving Environments 'Real Enough' for Exposure Therapy with Accident Victims? An Explorative Study." Cyber Psychology \& Behavior 8 (6): 532-537.

Wiederhold, B. K., A. H. Bullinger, and M. D. Wiederhold. 2006. "Advanced Technologies in Military Medicine." In Novel Approaches to the Diagnosis and Treatment of Posttraumatic Stress Disorder, edited by M. J. Roy, 148160. Amsterdam: IOS Press.

Witmer, B. G., and M. J. Singer. 1998. "Measuring Presence in Virtual Environments: A Presence Questionnaire." Presence: Teleoperators and Virtual Environments 7 (3): 225-240.

Wolpe, J. 1958. Psychotherapy by Reciprocal Inhibition. Stanford, CA: Stanford University Press. 


\section{Appendix}

Table A1. The implemented VR stressors and the main task.

\begin{tabular}{|c|c|c|}
\hline $\begin{array}{l}\text { The driving stressors identified } \\
\text { from the requirement analysis }\end{array}$ & $\begin{array}{c}\text { The implemented VR stressors in the } \\
\text { system }\end{array}$ & How it works in the VR environment \\
\hline \multicolumn{3}{|c|}{ Active stressors: Participants can respond to them when they occur } \\
\hline $\begin{array}{l}\text { A Baby crying } \\
\text { New family member } \\
\text { The need to keep a constant eye on where } \\
\text { the kids are and what they are doing } \\
\text { Experiencing high levels of noise }\end{array}$ & $\begin{array}{l}\text { Looking after a newborn baby } \\
\text { (loud crying baby) }\end{array}$ & $\begin{array}{l}\text { A model of newborn baby can be triggered to cry loudly in the } \\
\text { environment. The baby is located in a baby crib in the child's room. } \\
\text { The crying sound is a 3D sound that can be affected by distance and } \\
\text { rotation. The participant (parent in the VR environment) can respond } \\
\text { and interact with the baby by holding it in an animated sequence }\end{array}$ \\
\hline
\end{tabular}

C Too many interruptions Experiencing high levels of noise Too many things to do Too many things to do at once
Telephone (loud telephone ringing) toddler being in dangerous situations)

Too many interruptions

Too many things to do

Too many things to do at once

Too many responsibilities
D Too many interruptions Experiencing high levels of noise Too many things to do Too many things to do at once

E Too many interruptions Experiencing high levels of noise Too many things to do Too many things to do at once

F Unsatisfactory housing conditions Housework

Overloaded with family responsibilities Continually cleaning up messes of toys or food

Too many things to do Too many things to do at once Too many responsibilities

Passive stressors: Do not require a response from participants

$\mathrm{G}$ Troublesome neighbours Too many interruptions Experiencing high levels of noise

$\mathrm{H}$ Experiencing high levels of noise

I Experiencing high levels of noise. Too many interruptions

J All 13 identified stressors

\section{Neighbours' noise (loud argument and loud party)}

Street noise (loud siren)

Pet noise (loud dogs barking and cats meowing)

A combination of all the implemented stressors
The toddler stressor can be triggered to be in three different dangerous situations: trying to reach a knife on the kitchen ground, climbing and standing on a baby-feeding high chair, and trying to reach an open medication bottle. Participants were exposed to all three situations at different stages during the session (beginning, middle, or end of the session). The participant can respond and interact with the three situations: taking the knife and placing it in a knife block on the kitchen table, taking the child down from the high chair, and taking and closing the medication bottle and then putting it on a high, safe shelf out of reach of the toddler. The toddler was fully rigged and animated with facial expressions. The animation was a natural movement of the body parts in the same location. A 3D voice was also attached to the toddler to simulate a child talking. These features were implemented in an attempt to increase the sense of presence

A very loud telephone ringing with a $3 D$ tone located in the living room. The experimenter can trigger the phone and choose one of three possible recorded calls (i.e. family call, friend call, and telemarketer call). The same call was used with all participants in this experiment (family call). The phone calls were designed in a simple way as continuous talking. The scenarios of the calls developed to get the attention of the participant by expressing certain styles such as anger, excitement, or annoyance. The participant can respond to the phone ringing by answering the phone, which shows an animated sequence for the phone moving towards the participant's left ear, then the recorded call starts

The tone of the cell phone is very loud and in 3D. The cell phone is located in the main bedroom and follows similar features/process of the telephone. Only the recorded calls are different (IT support call, insurance call, and bank customer's service call). The same insurance call was used with all participants in the experiment

A loud doorbell that can be triggered for any period needed.

Participants respond by opening the door in which they find a post box left at the doorstep. They then take it and place it on the kitchen table

This was considered as a stressor because very messy/untidy places can stress some people.

Also, it was used as a main task in the VR environment. The action taken towards this situation was tidying up by picking up the task items and then placing them in the appropriate places

A neighbour couple arguing and fighting with each other loudly. Also, gathering at a neighbour's house, partying loudly

A fire fighter's car with loud siren noise passing in front the house A group of dogs barking and fighting each other loudly just behind the participant's house fence, and cats meowing loudly inside the house All stressors are triggered at once to create more demand and greater level of stress 